\title{
The Atacama Cosmology Telescope: A Search for Planet 9
}

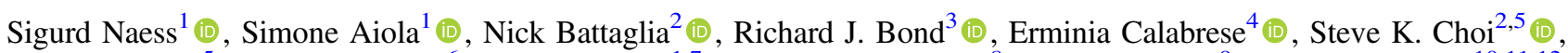

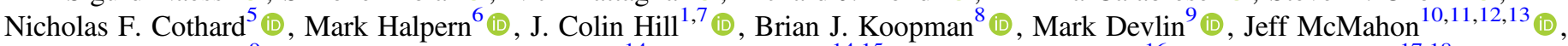

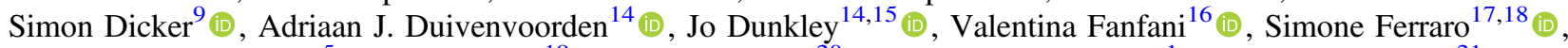
Patricio A. Gallardo ${ }^{5}$ (10), Yilun Guan ${ }^{19}$ (1), Dongwon $\operatorname{Han}^{20}$ (1), Matthew Hasselfield ${ }^{1}$ (1) , Adam D. Hincks ${ }^{21}$ (1), Kevin Huffenberger ${ }^{22}$ (1), Arthur B. Kosowsky ${ }^{19}$ (i), Thibaut Louis ${ }^{23}$ (i), Amanda Macinnis ${ }^{20}$, Mathew S. Madhavacheril ${ }^{24}$ (1), Federico Nati ${ }^{16}$ (i) , Michael D. Niemack ${ }^{2,5,25}$ (10), Lyman Page ${ }^{14}$ (i), Maria Salatino ${ }^{26}$ (iD), Emmanuel Schaan ${ }^{17,18}$ (1), John Orlowski-Scherer ${ }^{9}$ (1) , Alessandro Schillaci ${ }^{27}$ (1), Benjamin Schmitt ${ }^{28}$, Neelima Sehgal ${ }^{20}$ (1) , Cristóbal Sifón $^{29}$ (i) , Suzanne Staggs ${ }^{14}$ (i), Alexander Van Engelen ${ }^{30}$, and Edward J. Wollack ${ }^{31}$ (i)

${ }^{1}$ Center for Computational Astrophysics, Flatiron Institute, New York, NY 10010, USA

${ }^{2}$ Department of Astronomy, Cornell University, Ithaca, NY 14853, USA

${ }^{3}$ Canadian Institute for Theoretical Astrophysics, 60 St. George Street, University of Toronto, Toronto, ON, M5S 3H8, Canada

${ }^{4}$ School of Physics and Astronomy, Cardiff University, The Parade, Cardiff, Wales, CF24 3AA, UK ${ }^{5}$ Department of Physics, Cornell University, Ithaca, NY 14853, USA

${ }^{6}$ Department of Physics and Astronomy, University of British Columbia, Vancouver, BC, V6T 1Z4, Canada

${ }^{7}$ Department of Physics, Columbia University, New York, NY 10027, USA

${ }^{8}$ Department of Physics, Yale University, New Haven, CT 06520, USA

${ }^{9}$ Department of Physics and Astronomy, University of Pennsylvania, 209 South 33rd Street, Philadelphia, PA 19104, USA

${ }^{10}$ Kavli Institute for Cosmological Physics, University of Chicago, Chicago, IL 60637, USA

${ }^{11}$ Department of Astronomy and Astrophysics, University of Chicago, Chicago, IL 60637, USA

${ }_{13}$ Department of Physics, University of Chicago, Chicago, IL 60637, USA

${ }^{13}$ Enrico Fermi Institute, University of Chicago, Chicago, IL 60637, USA

14 Joseph Henry Laboratories of Physics, Jadwin Hall, Princeton University, Princeton, NJ 08544, USA

15 Department of Astrophysical Sciences, Peyton Hall, Princeton University, Princeton, NJ 08544, USA

${ }^{16}$ Department of Physics, University of Milano-Bicocca, Piazza della Scienza, I-3-20126 Milano (MI), Italy Lawrence Berkeley National Laboratory, Berkeley, CA 94720, USA

18 Berkeley Center for Cosmological Physics, University of California, Berkeley, CA 94720, USA

${ }_{20}^{19}$ Department of Physics and Astronomy, University of Pittsburgh, Pittsburgh, PA 15260, USA

${ }^{20}$ Physics and Astronomy Department, Stony Brook University, Stony Brook, NY 11794, USA

${ }^{21}$ Department of Astronomy and Astrophysics, University of Toronto, 50 St. George Street, Toronto, ON M5S 3H4, Canada

22 Department of Physics, Florida State University, Tallahassee FL 32306, USA

${ }^{23}$ Université Paris-Saclay, CNRS/IN2P3, IJCLab, F-91405 Orsay, France

${ }^{24}$ Perimeter Institute for Theoretical Physics, 31 Caroline Street N, Waterloo ON N2L 2Y5 Canada

${ }^{25}$ Kavli Institute at Cornell for Nanoscale Science, Cornell University, Ithaca, NY 14853, USA

${ }^{26}$ Physics Department, Stanford University Kavli Institute for Particle Astrophysics and Cosmology (KIPAC) Stanford, CA, USA

${ }^{27}$ Department of Physics, California Institute of Technology, Pasadena, CA 91125, USA

${ }^{28}$ Harvard-Smithsonian Center for Astrophysics, USA

${ }^{29}$ Instituto de Física, Pontificia Universidad Católica de Valparaíso, Casilla 4059, Valparaíso, Chile

${ }^{30}$ School of Earth and Space Exploration, Arizona State University, Tempe, AZ 85287, USA

${ }^{31}$ NASA/Goddard Space Flight Center, Greenbelt, MD 20771, USA

Received 2021 May 11; revised 2021 August 30; accepted 2021 August 31; published 2021 December 23

\begin{abstract}
We use Atacama Cosmology Telescope (ACT) observations at 98 GHz (2015-2019), 150 GHz (2013-2019), and $229 \mathrm{GHz}$ (2017-2019) to perform a blind shift-and-stack search for Planet 9. The search explores distances from $300 \mathrm{au}$ to $2000 \mathrm{au}$ and velocities up to 6.3 per year, depending on the distance ( $r$ ). For a 5 Earth-mass Planet 9 the detection limit varies from 325 au to 625 au, depending on the sky location. For a 10 Earth-mass planet the corresponding range is 425 au to 775 au. The predicted aphelion and most likely location of the planet corresponds to the shallower end of these ranges. The search covers the whole 18,000 square degrees of the ACT survey. No significant detections are found, which is used to place limits on the millimeter-wave flux density of Planet 9 over much of its orbit. Overall we eliminate roughly $17 \%$ and $9 \%$ of the parameter space for a 5 and 10 Earth-mass Planet 9, respectively. These bounds approach those of a recent INPOP19a ephemeris-based analysis, but do not exceed it. We also provide a list of the 10 strongest candidates from the search for possible follow-up. More generally, we exclude (at 95\% confidence) the presence of an unknown solar system object within our survey area brighter than 4-12 mJy (depending on position) at $150 \mathrm{GHz}$ with current distance $300 \mathrm{au}<r<600$ au and heliocentric angular velocity $1.5 \mathrm{yr}^{-1}<v \cdot \frac{500 \mathrm{au}}{r}<2$ !! $3 \mathrm{yr}^{-1}$, corresponding to low-to-moderate eccentricities. These limits worsen gradually beyond $600 \mathrm{au}$, reaching 5-15 mJy by $1500 \mathrm{au}$.
\end{abstract}

Unified Astronomy Thesaurus concepts: Solar system planets (1260); Millimeter astronomy (1061); Sky surveys (1464) 


\section{Introduction}

The existence of "Planet 9," a large (mass $M \sim 5-10 M_{\oplus}$ ) and very distant (semimajor axis $a \sim 400-800 \mathrm{au}$ ) new planet in the solar system, has recently been proposed as an explanation for the observed clustering of orbits of the highest-perihelion objects in the detached Kuiper Belt, the detachment of perihelion of Sednoids, and the generation of high-inclination extreme trans-Neptunian objects and retrograde centaurs (Batygin \& Brown 2016; Batygin et al. 2019; hereafter B16 and B19). While the significance of these effects is unclear because of the presence of large observational biases (Shankman et al. 2017; Bernardinelli et al. 2020; Napier et al. $2021),{ }^{32}$ the hypothesis has still gathered considerable interest.

Most new solar system objects are discovered in optical surveys via their reflected sunlight. At these wavelengths, Planet 9 would appear as a magnitude 19-24 object (depending on the size and distance and assuming an albedo between 0.4 and 1; B19, page 61): quite faint due to the $1 / r^{4}$ dependence of reflected sunlight, ${ }^{33}$ but still detectable by optical surveys like the Dark Energy Survey (DES), the Hyper-Suprime Cam survey (HSC), or the Legacy Survey of Space and Time (LSST).

The steep falloff of flux density with distance can be circumvented by observing at longer wavelengths, where thermal emission dominates. The heat budget of large objects far from the Sun is dominated by their gravitational contraction and residual heat of formation, resulting in a temperature that is approximately independent of their distance from the Sun. This leads to a much gentler $1 / r^{2}$ dependence. For sufficiently large distances this can partially compensate for, or even overcome, the resolution advantage enjoyed by optical surveys compared to those at millimeter or submillimeter wavelengths. Indeed, the best current limits on the existence of Saturn- or Jupiter-size trans-Neptunian objects (TNOs) is the Wide-field Infrared Survey Explorer (WISE), which observes in the 2.8-26 $\mu \mathrm{m}$ (11-110 THz) range. WISE has excluded the existence of a Saturn-size planet out to 28,000 au, and a Jupiter-size one out to 82,000 au (Luhman 2013). Sadly, these limits degrade very quickly with mass, partially because of a decrease in surface area, but more importantly because lower-mass planets cool down more quickly. For sufficiently low masses, the majority of the thermal emission would fall outside the WISE frequency range, and this is expected to be the case for typical atmospheric models (see Section 2). However, emission predictions in the 3-5 $\mu \mathrm{m}$ window are extremely model dependent, varying by four orders of magnitude. The brightest of these could be detectable by WISE. Meisner et al. (2018) report a nondetection of Planet 9 in WISE's $3.6 \mu \mathrm{m} \mathrm{W} 1$ band, limiting its W1 magnitude to $>16.7$ (flux density $<65 \mu \mathrm{Jy}$ ) at $90 \%$ confidence. For the most optimistic atmospheric models, this excludes a $10 M_{\oplus}$ Planet 9 up to $900 \mathrm{au}$, but for more typical cases WISE would not be sensitive to Planet 9's thermal radiation, motivating a search at lower frequencies.

Soon after Planet 9 was first proposed, Cowan et al. (2016; and later Baxter et al. 2018) suggested a search using Cosmic Microwave Background (CMB) telescopes operating in the 1-3 mm range. The only current CMB survey telescopes with a high enough resolution to have any hope of detecting a faint, unresolved object like Planet 9 are the South Pole Telescope (SPT;

\footnotetext{
32 https://findplanetnine.blogspot.com/2021/02/is-planet-nine-finallydead.html

${ }^{33}$ Here, $r$ is the object's current distance from the Sun. Technically the expression should be $1 /\left(r^{2} r_{\oplus}^{2}\right)$ where $r_{\oplus}$ is the distance from the Earth, but in the outer solar system $r \approx r_{\oplus}$.
}

Carlstrom et al. 2011) and the Atacama Cosmology Telescope (ACT; Fowler et al. 2007; Thornton et al. 2016), and of these only ACT covers the low ecliptic latitudes where Planet 9 might lurk.

ACT is a $6 \mathrm{~m}$ millimeter-wave telescope located at $5190 \mathrm{~m}$ altitude on Cerro Toco in the northern Chilean Andes. ACT began observations in 2008, and has been upgraded several times to add polarization support and increase its sensitivity and frequency coverage. ACT is currently surveying 18,000 square degrees of the sky in five broad bands roughly centered on $27,39,98,150$, and $229 \mathrm{GHz}$, though the first two were added too recently to be available for this analysis. We label these bands f030, f040, f090, f150, and f220, respectively.

The primary goal of the survey is to map the CMB, but the telescope's relatively high angular resolution of $2 ! 05 / 1 ! 40 /$ 0 !98 FHWM in the f090/f150/f220 bands, respectively, makes it capable of a large set of other science goals, including searches for galaxy clusters, active galactic nuclei, and transients. We here report on a search for Planet 9 using $7 \mathrm{yr}$ of ACT data collected from 2013 to 2019.

\section{Planet 9 in the ACT Bands}

Linder \& Mordasini (2016) and Fortney et al. (2016; henceforth F16) have investigated the radius, temperature, and luminosity of Planet 9, and found that the Sun has a minimal impact on its heat budget, and hence its physical properties do not depend on the planet's distance from the Sun. They do, however, depend considerably on both its mass and internal composition, for which the papers consider several models. Linder \& Mordasini (2016) and F16 agree on the most likely models, but differ somewhat on which less likely variants they investigate. ${ }^{34}$ We will here follow F16 due to their good coverage of both the $5 M_{\oplus}$ and $10 M_{\oplus}$ scenarios.

Their nominal scenario has an $\mathrm{H} / \mathrm{He}$ envelope making up $10 \%$ of the planet's mass, with the remainder being mostly a 2:1 mix of ice and rock. For this composition they find that the most favored $5 M_{\oplus}$ scenario of B19 results in a radius of $2.94 R_{\oplus}$, a temperature of $42.2 \mathrm{~K}$ and a featureless blackbody spectrum below $8 \mathrm{THz} .{ }^{35}$ For a fiducial distance of $500 \mathrm{au}$, this results in a flux density of $2.3 \mathrm{mJy}, 5.3 \mathrm{mJy}$, and $11 \mathrm{mJy}$ in the three ACT bandpasses f090, f150, and f220. For the $10 M_{\oplus}$ scenario, which is near the upper end of the possible mass range, the corresponding numbers are $R=3.46 R_{\oplus}, T=48.3 \mathrm{~K}$, and a flux density of 3.7/8.5/18 mJy at f090/f150/f220. These numbers vary by $10 \%-50 \%$ depending on the compositionsee Table $1 .{ }^{36}$ Depending on Planet 9's exact orbit, its current distance could vary from about 300 au to $1200 \mathrm{au}$, but due to the radius and temperature being independent of the distance from the Sun, this simply rescales the flux densities as $1 / r^{2}$.

The expected distance to Planet 9 is correlated with its mass, since a more massive planet has to be further away to avoid having too large of an effect on the orbits of other transNeptunian objects. A $5 M_{\oplus}$ Planet 9 would have an expected

\footnotetext{
${ }^{34}$ Linder \& Mordasini (2016) include a scenario where Planet 9 is a $10 M_{\oplus}$ super-Earth without any significant atmosphere. This case has $R=1.9 R_{\oplus}$ and $T=38 \mathrm{~K}$, making it roughly 4 times as faint as the nominal case, resulting in ACT distance limits being half as far.

35 Note: F16 cautions that while their framework fits Neptune well, it overestimates Uranus's temperature, and they cannot exclude that this could be the case for Planet 9 too.

${ }^{36}$ This ignores the small loss of flux density that comes from the planet blocking the $2.725 \mathrm{~K} \mathrm{CMB}$ monopole. This leads to a $2.6 \% / 1.4 \% / 0.6 \%$ loss of flux density at $\mathrm{f} 090 / \mathrm{f} 150 / \mathrm{f} 220$, which is negligible compared to the uncertainty on Planet 9's physical properties.
} 
Table 1

Potential Radii and Temperatures for a $5 M_{\oplus}$ and $10 M_{\oplus}$ Planet 9 from F16

\begin{tabular}{|c|c|c|c|c|c|c|c|}
\hline $\begin{array}{l}\text { Mass } \\
\left(M_{\oplus}\right)\end{array}$ & $\begin{array}{c}\text { Radius } \\
\left(R_{\oplus}\right)\end{array}$ & $\begin{array}{l}\text { Temperature } \\
\text { (K) }\end{array}$ & Band & $\begin{array}{c}\text { Flux @ } @ 500 \text { au } \\
\text { (mJy) }\end{array}$ & $\begin{array}{l}\text { ACT Depth } \\
\text { (mJy) }\end{array}$ & $\begin{array}{l}\text { FWHM } \\
\text { (arcmin) }\end{array}$ & $\begin{array}{l}\text { Freq. } \\
(\mathrm{GHz})\end{array}$ \\
\hline \multirow{3}{*}{5} & \multirow{3}{*}{$4.12 / 2.94 / 2.71$} & \multirow{3}{*}{$36.7 / 42.2 / 38.9$} & f090 & $3.9 / 2.3 / 1.8$ & $1.0-2.1$ & 2.05 & 98 \\
\hline & & & $\mathrm{f} 150$ & $8.9 / 5.3 / 4.1$ & $1.0-2.2$ & 1.40 & 150 \\
\hline & & & $\mathrm{f} 220$ & $18 / 11 / 8.5$ & $4.1-8.4$ & 0.98 & 229 \\
\hline \multirow[b]{2}{*}{10} & \multirow[b]{2}{*}{$5.09 / 3.46 / 3.16$} & \multirow[b]{2}{*}{$40.3 / 48.3 / 45.1$} & f090 & $6.6 / 3.7 / 2.9$ & $1.0-2.1$ & 2.05 & 98 \\
\hline & & & f150 & $15 / 8.5 / 6.6$ & $1.0-2.2$ & 1.40 & 150 \\
\hline
\end{tabular}

Note. The three slash-separated entries correspond to three planet types described in their Table 1 . The central one is the nominal case with a 2:1 ice:rock core surrounded by an $\mathrm{H} / \mathrm{He}$ envelope. The leftmost entries are for a case with a larger $\mathrm{H} / \mathrm{He}$ envelope and the rightmost entries are for the ice-poor case, for which the core is 1:2 ice:rock by mass. The corresponding flux density in the three ACT frequency bands for these cases is given in the flux density column, and compared to the ACT point-source sensitivity, which is about 1-2 mJy as seen in the sixth column. The first/last number in the range of ACT depth is the $10 \% / 90 \%$ quantile over the 18,000 square degrees that ACT covers. The maps are deep enough compared to the expected Planet 9 flux density that a search is worthwhile. Also shown are the ACT beam size and central frequency in each band.

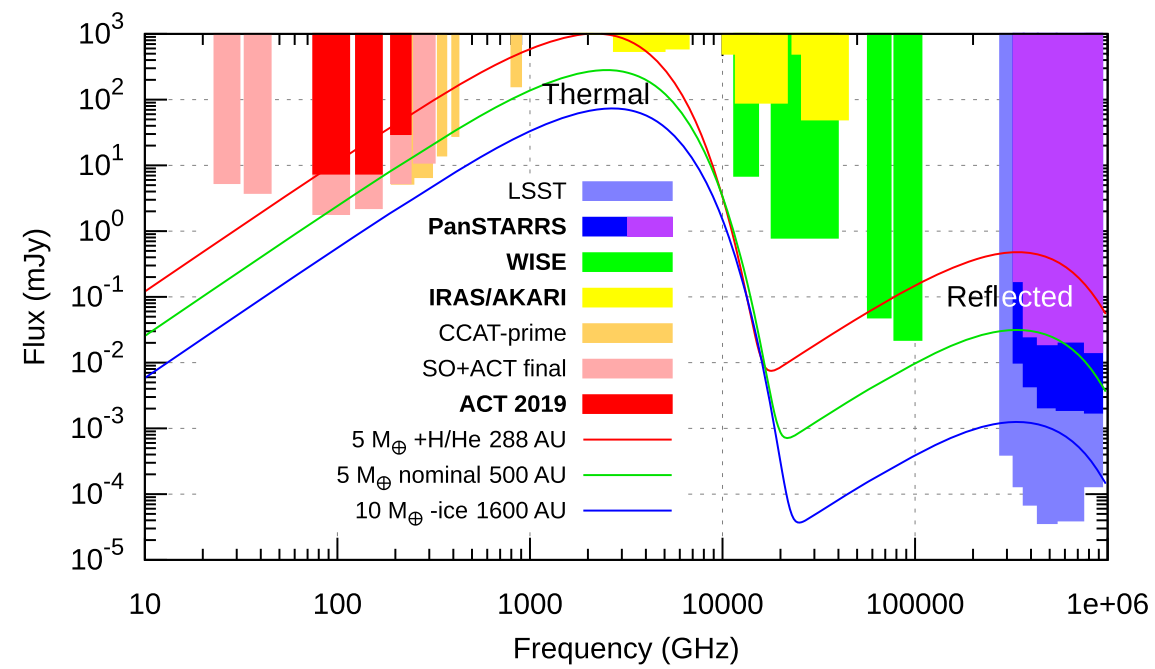

Figure 1. The potential Planet 9 spectra compared to the $5 \sigma$ detection limit of current and upcoming wide-area surveys. Red curve: high-brightness scenario: a $5 M_{\oplus}$ Planet 9 with a heavy $\mathrm{H} / \mathrm{He}$ envelope at a perihelion of 288 au. Green curve: a more moderate scenario with a light $\mathrm{H} / \mathrm{He}$ envelope and a 2:1 ice:rock ratio at 500 au, still with $5 M_{\oplus}$. Blue curve: low-brightness scenario of a $10 M_{\oplus}$ Planet 9 with a light $\mathrm{H} / \mathrm{He}$ envelope and a 1:4 ice:rock ratio at an aphelion of 1160 au. All scenarios assume unit emissivity blackbody spectra, but differ in the planet radius and release and transport of internal gravitational energy; see F16 Table 1. ACT 2019 is the data set used in this paper, while SO+ACT final is the expected combined Simons Observatory (SO Collaboration 2019) + ACT data after both surveys finish. The others are CCAT-prime (Choi et al. 2020), IRAS (W.G. \& J.I.S., 1986), AKARI (Ishihara et al. 2010; Yamamura et al. 2010), WISE (AllWISE 2013; Schlafly et al. 2019), Pan-STARRS (Chambers et al. 2016), and LSST (Ivezić et al. 2019). Future surveys are shown with a thinner font and less intense color in the legend. For Pan-STARRS both the full depth (blue) and the transient search depth (purple; Pan-STARRS 2015) are shown. The double blackbody approximation used here may be inaccurate in the range 8-400 THz because of atmospheric features (F16, Figure 1). The sensitivities shown are typical values for each survey, and do not attempt to compensate for the surveys' different sky coverage. Depth variations inside each survey and the effect of the large parameter space of a blind search for a moving object are ignored.

semimajor axis $a \sim 500$ au and an eccentricity of $0.1 \lesssim e \lesssim 0.3$, while at $10 M_{\oplus}$ the best-fit semimajor axis and eccentricity are $a \sim 700$ au and $0.3 \lesssim e \lesssim 0.5$ (B19, Figure 15$).{ }^{37}$ At frequencies $<2.5 \mathrm{THz}$, this increased distance mostly cancels the increased luminosity of a more massive planet, making ACT's prospect for detecting an object like Planet 9 only moderately sensitive to its mass. ${ }^{38}$ The planet's inclination is predicted to be moderate, $i<30^{\circ}$, with $i \approx 20^{\circ}$ preferred.

\footnotetext{
${ }^{37}$ Batygin \& Brown (2021) give somewhat higher eccentricities, but do not quantify by how much, and it is limited by how much the eccentricity can be increased without running afoul of the bounds in B19. Due to the lack of concrete numbers in this newer work we use the B19 bounds here.

${ }^{38}$ This is in contrast to $2.5 \mathrm{THz}<\nu<20 \mathrm{THz}$ where small changes in mass lead to big changes in detectability because of the steep fall of the blackbody spectrum here, and $\nu>20 \mathrm{THz}$ where the $1 / r^{4}$ dependence of reflected sunlight makes a smaller, closer planet much easier to detect.
}

To see if ACT has any chance of detecting this signal, let us compare it to ACT's sensitivity to stationary point sources. This varies by position in the map but the $10 \%-90 \%$ quantile range is about $1-2 \mathrm{mJy}$ at $\mathrm{f090}$ and $\mathrm{f150}$, and $4-8 \mathrm{mJy}$ at f220 ${ }^{39}$ Hence, if Planet 9 were stationary at 500 au, we could expect to detect it at $2.3-11 \sigma$ for the $5 M_{\oplus}$ case when combining the three ACT bands. This is not high enough to guarantee a discovery, especially considering that Planet 9 could be at a larger distance than $500 \mathrm{au}$, but it is high enough that a search is worthwhile.

Figure 1 compares the brightest/medium/faintest expected Planet 9 spectra (as inferred from the range of possible orbits from B19 and of physical properties from F16) to the sensitivity of ACT and other current and future wide-area surveys. Despite

\footnotetext{
${ }^{39}$ For comparison, the same quantile range for Planck $143 \mathrm{GHz}$ is $29-41 \mathrm{mJy}$.
} 
WISE's impressive bounds on Saturn- and Jupiter-size TNOs, it is not very sensitive to smaller, and therefore colder, objects like Planet 9. The most sensitive current data set that covers most/all of Planet 9's orbit is therefore Pan-STARRS. At its full depth of about magnitude 23, Pan-STARRS has a flux density limit of roughly $2 \mu \mathrm{Jy}$, but this degrades to around $20 \mu \mathrm{Jy}(\mathrm{mag} \approx 21)$ if the search is limited to the depth of the Pan-STARRS transient search (Pan-STARRS 2015, B19). Both WISE and Pan-STARRS have reduced sensitivity near the Galactic plane because of confusion. For the medium brightness case, ACT's typical depth could expect a borderline detection, similar to the Pan-STARRS transient search and a bit better than WISE.

\section{The ACT Data Sets}

The data sets used in this analysis are identical to those used in Naess et al. (2020), except for the inclusion of one more season of data (2019), and the exclusion of the Planck and ACT MBAC data sets because of their low resolution and low sky coverage, respectively. This represents $7 \mathrm{yr}$ and $140 \mathrm{~TB}$ of data, of which $81 \%$ was collected since the AdvACT camera (Ho et al. 2017; Choi et al. 2018) became operational in 2017 (i.e., after ACT Data Release 4). ${ }^{40}$ See Appendix D for details.

\section{Search Methodology}

\subsection{Blink Comparison Will Not Work}

The most common way to discover solar system objects is to look for objects that have moved between two different exposures of the same patch of sky. This method is fast, but is limited by the depth of each image, since the object needs to be independently detected in both. This depth can be improved with longer exposures, but this is limited by the angular velocity of the object itself. Integrating longer than the time it takes the object to move by the size of the beam will just smear it out without any further gains in signal-to-noise ratio $(\mathrm{S} / \mathrm{N})$. This is the regime ACT is in for Planet 9.

For ACT sky coverage and sensitivity, it would take 3-4 yr of observations just to have a chance of detecting a Planet 9like object that was not moving in the sky. By Kepler's laws, a planet with semimajor axis $a$, eccentricity $e$, and current solar distance $r$ will have a Sun-centered angular speed of

$$
v=1.932 \mathrm{yr}^{-1} \cdot \sqrt{\frac{a}{500 \mathrm{au}}}\left(\frac{r}{500 \mathrm{au}}\right)^{-2} \sqrt{1-e^{2}} .
$$

At the same time, the Earth's orbit sweeps out a yearly parallax ellipse with a semimajor axis of

$$
\theta_{\pi}=\frac{\mathrm{au}}{r}=6.875 \cdot \frac{500 \mathrm{au}}{r}
$$

corresponding to a maximum angular speed of

$$
v_{\pi}=\frac{2 \pi \theta_{\pi}}{\mathrm{yr}}=43.20 \mathrm{yr}^{-1} \cdot \frac{500 \mathrm{au}}{r} .
$$

For comparison, ACT has an angular resolution of $2.05^{\prime} / 1.40^{\prime} /$ $0.98^{\prime}$ FWHM at f090/f150/f220, respectively. To avoid excessive smearing we need $\left(v_{\pi}+v\right) \Delta t \approx v_{\pi} \Delta t \ll$ FWHM. For the smallest beam (f220) and a closest possible distance of $r_{\min }=300 \mathrm{au}$, this gives us $\Delta t \ll 5$ days. With 5 days of integration time and the

\footnotetext{
${ }^{40}$ Split by frequency, that is $37 / 72 / 17$ TB at $\mathrm{f} 090 / \mathrm{f} 150 / \mathrm{f} 220$, of which $93 \% /$ $71 \% / 100 \%$ was collected since 2017 .
}

current ACT survey strategy the expected Planet $9 \mathrm{~S} / \mathrm{N}$ would be $\sim 1$, more than 5 times too low for a detection, or more than 25 times too low in terms of observing time!

\subsection{Shift and Stack}

The smearing could be eliminated if one knew the orbit of the object one was looking for, since that would allow one to shift each exposure to track the object as it moves across the sky. In practice, while the Planet 9 hypothesis makes some predictions about its orbit, they are far too vague to allow for simple tracking like this. However, with enough computational resources it is possible to loop through every reasonable orbit, make a shifted stack of individual short exposures using that orbit, and then look for objects in the resulting image. This is the shift-and-stack algorithm, and has been used to successfully detect objects below the single-exposure sensitivity limit (Gladman et al. 1998; Bernstein et al. 2004; Holman et al. 2018).

Planet 9's orbit is characterized by its six orbital elements: semimajor axis $a$, eccentricity $e$, inclination $i$, longitude of ascending node $\Omega$, argument of periapsis $\omega$, and true anomaly $\nu$. However, because of its large distance and corresponding slow motion, it is sufficient for us to consider its motion to be drifting linearly on the sky, modulated by parallax. This gives us the following five free parameters:

1. The heliocentric R.A. $(\alpha)$ and decl. $(\delta)$ of the planet at a reference time $t_{0}$.

2. The horizontal and vertical components of the heliocentric angular velocity $\boldsymbol{v}=\left[v_{x}, v_{y}\right]$. We define these in the local tangent plane, such that $\alpha_{\mathrm{obs}}=\alpha+v_{x}\left(t-t_{0}\right) / \cos \delta_{0}$ and $\delta_{\text {obs }}=\delta+v_{y}\left(t-t_{0}\right)$.

3. The planet's current distance from the Sun, $r$, which we treat as constant in time.

With these, the shift-and-stack algorithm takes the following general form:

1. Split the data into chunks with duration $\Delta t$, and make a sky map of each.

2. For each reasonable value of $r, v_{x}$, and $v_{y}$, use these with the time $t$ of each map to shift them according to their constant heliocentric angular velocity and parallactic motion, and stack them to produce a combined map.

3. Use a filter matched to the noise and signal properties to look for point sources in each combined map.

We will go through the details of this process in the following sections.

\subsection{Mapping and the Matched Filter}

$$
\text { 4.3.1. The Sky Maps }
$$

ACT observes the sky by sweeping backwards and forwards in azimuth while the sky drifts past. As it does so, the temperature registered by the detectors is read out hundreds of times per second, forming a vector of time-ordered data $d$. We model $d$ as

$$
d=P m+n,
$$

where $m$ is the (beam-convolved, pixelated ${ }^{41}$ ) sky in $\mu \mathrm{K} \mathrm{CMB}$ temperature units, $P$ is a response matrix that encodes the telescope's pointing as a function of time, and $n$ is instrumental

\footnotetext{
${ }^{41}$ We use 0.5 pixels in a Plate Carreé projection in equatorial coordinates. This is later downsampled to $1^{\prime}$ pixels (see Section 4.4).
} 
and atmospheric noise, which we model as Gaussian covariance $N$. The maximum-likelihood estimate for $m$ given $d$ is

$$
\begin{aligned}
\hat{m} & =\left(P^{T} N^{-1} P\right)^{-1} P^{T} N^{-1} d \\
M & =\left(P^{T} N^{-1} P\right)^{-1}
\end{aligned}
$$

Here, $M$ is the noise covariance matrix of the estimator $\hat{m}$.

\subsubsection{The Matched Filter}

To look for point sources in $\hat{m}$ we start by assuming that all sources are far enough apart that they can be considered in isolation. Our data model for a map containing a single point source in some pixel $p$ is then

$$
\hat{m}=R Q s_{p}+u
$$

where $s_{p}$ is the point-source flux density in pixel $p$ in mJy at a reference frequency $\nu_{0}=150 \mathrm{GHz}$, and $Q_{i}=\delta_{i p}$ is a vector that is unity at the source location in pixel $p$ and zero elsewhere. It takes us from just a single flux density value to a map with that value in a single pixel.

$R=B g\left(\nu_{0}, \nu\right) f(\nu) A_{p}$ is a response matrix that takes us from that map to beam-convolved $\mu \mathrm{K}$ at the observed frequency. Here, $B$ is the instrument beam normalized to have a pixelspace integral of one, $g\left(\nu_{0}, \nu\right)$ is the conversion factor from flux density at the reference frequency $\nu_{0}$ to the observed frequency $\nu, f(\nu)$ is the conversion from flux density in mJy to beamconvolved peak height in $\mu \mathrm{K}$, and $A_{p}$ is pixel area in steradians. Since we expect Planet 9 to be a blackbody with temperature $T \approx 40 \mathrm{~K}$, we have $g\left(\nu_{0}, \nu\right)=b(\nu, T) / b\left(\nu_{0}, T\right)$, where

$$
b(\nu, T)=\frac{2 h \nu^{3}}{c^{2}} \frac{1}{\exp \left(\frac{h \nu}{k_{B} T}\right)-1}
$$

is the Planck law for surface brightness $b$; and $\mathrm{d}^{42}$

$$
\begin{aligned}
f(\nu) & =\left(\frac{2 x^{4} k_{B}^{3} T_{\mathrm{CMB}}^{2}}{h^{2} c^{2}} \frac{1}{4 \sinh (x / 2)^{2}} 10^{23} A_{b}\right)^{-1} \\
x & =\frac{h \nu}{k_{B} T_{\mathrm{CMB}}} .
\end{aligned}
$$

Finally, $u$ is the noise in $\hat{m}$ and has a covariance matrix $U$. For the purposes of point-source detection, $u$ consists of everything in $\hat{m}$ that is not the point source, which includes both the instrumental and atmospheric noise described by the $M$ covariance matrix from before, but also the CMB, Cosmic Infrared Background (CIB), Galactic dust, etc.

Given this model for $\hat{m}$, the maximum-likelihood estimate for the point-source flux density at the reference frequency is

$$
\begin{gathered}
\hat{s}_{p}=\rho_{p} / \kappa_{p}, \rho_{p}=Q^{T} R^{T} U^{-1} \hat{m} \\
\sigma_{\hat{s}_{p}}=1 / \sqrt{\kappa_{p}}, \kappa_{p}=Q^{T} R^{T} U^{-1} R Q
\end{gathered}
$$

Here, $\sigma_{\hat{s}_{p}}$ is the standard deviation of $\hat{s}_{p}, \kappa_{p}$ is the corresponding inverse variance, and $\rho_{p}$ is the inverse variance weighted flux density.

\footnotetext{
42 In the expression for $f(\nu), A_{b}^{-1}$ converts from mJy to $\mathrm{mJy} \mathrm{sr}^{-1}, 10^{-23}$ converts from mJy sr${ }^{-1}$ to $\mu \mathrm{W} \mathrm{m}{ }^{-2} \mathrm{~Hz}^{-1} \mathrm{sr}^{-1}$, and the rest is the derivative of the Planck law evaluated at $T=T_{\mathrm{CMB}}$, and converts to linearized $\mathrm{CMB}$ units in $\mu \mathrm{K}$.
}

So far we have only estimated the point-source flux density in some pixel $p$. But since we do not a priori know where on the sky the planet could be, we need to estimate the flux density in every pixel, resulting in the flux density sky map $\hat{s}$ and corresponding uncertainty $\sigma_{\hat{s}}$ given by: ${ }^{43}$

$$
\begin{aligned}
\hat{s} & =\rho / \kappa, \rho=R^{T} U^{-1} \hat{m} \\
\sigma_{\hat{s}} & =1 / \sqrt{\kappa}, \kappa=\operatorname{diag}\left(R^{T} U^{-1} R\right)
\end{aligned}
$$

where the division is done pixel by pixel. The corresponding $\mathrm{S} / \mathrm{N}$ is

$$
\mathrm{S} / \mathrm{N}=\boldsymbol{s} / \sigma_{\mathrm{s}}=\rho / \sqrt{\kappa}=\frac{R^{T} U^{-1} \hat{m}}{\sqrt{\operatorname{diag}\left(R^{T} U^{-1} R\right)}}
$$

which we recognize as the matched filter for $\hat{m}$. This $\mathrm{S} / \mathrm{N}$ map is what one would usually use for object detection, e.g., by identifying peaks with $\mathrm{S} / \mathrm{N}>5$. As we shall see in Section 4.7 the shift-and-stack parameter search complicates this, but the general idea stays the same.

\subsubsection{Stacking}

If we have multiple estimates $\left\{\boldsymbol{s}_{i}\right\}$ built from independent chunks of data, such as the few-day chunks we will use in the shift-and-stack algorithm, these combine straightforwardly: ${ }^{44}$

$$
\begin{aligned}
\rho_{\mathrm{tot}} & =\sum_{i} \rho_{i}, \kappa_{\mathrm{tot}}=\sum_{i} \kappa_{i} \\
\boldsymbol{s}_{\mathrm{tot}} & =\rho_{\mathrm{tot}} / \kappa_{\mathrm{tot}}, \sigma_{\mathrm{s}_{\mathrm{tot}}}=1 / \sqrt{\kappa_{\mathrm{tot}}} .
\end{aligned}
$$

Sadly, the presence of the same CMB, CIB, etc. in each chunk of data breaks the assumption of independence that this expression builds on. It would be possible to build a more complicated expression that takes this into account, but given the computationally expensive parameter search we perform we need the stacking operation to be as fast and simple as possible. Thankfully we can eliminate these correlated components by simply subtracting the time-averaged mean of the sky from each chunk of data.

\subsubsection{Mean Sky Subtraction}

We can avoid the complications of the CMB, CIB, etc. acting as correlated noise common to the data chunks by subtracting a high $\mathrm{S} / \mathrm{N}$ estimate of the mean sky from each chunk of data before mapping it. This eliminates any static part of the sky such as the CMB, CIB, Galactic emission, etc. (including any we do not know about), and leaves only timedependent signals such as the planet we are looking for, as well as variable point sources (which can be masked) and transients (which are rare enough that we can ignore them). The cost is a small increase in the noise if the mean sky model is not noise free, and a partial subtraction of the signal itself that must be estimated and corrected for. For this search we use the ACT +Planck combined maps described in Naess et al. (2020), but extended to include the 2019 season of data.

Aside from letting us stack using Equation (12), mean sky subtraction has the effect of removing all but the instrumental and atmospheric noise from the individual sky maps, and hence

\footnotetext{
${ }^{43}$ Since $Q / Q^{T}$ just picks out an individual row of the quantity it is applied to, $\rho_{p}$ is just element $p$ of the vector $R^{T} U^{-1} \hat{m}$ and $\kappa_{p}$ is just element $p$ along the diagonal of $R^{T} U^{-1} R$

${ }^{44}$ Unlike the previous section, where, e.g., $\rho_{p}$ was the value in a single pixel, here each $\rho_{i}$ is a whole map.
} 
the matched filter noise covariance matrix $U$ reduces to $M$. Inserting this into Equation (10) we get:

$$
\rho=R^{T}{\overbrace{P^{T} N^{-1} d}^{\text {rhs }}}^{\text {ro }}
$$

The part labeled "rhs" is a map that is much cheaper to compute than $\hat{m}$ because it avoids the expensive inversion $\left(P^{T} N^{-1} P\right)^{-1}=M$ that must usually be done using iterative methods like conjugate gradients. ${ }^{45}$ That leaves us with $\kappa$, which we approximate as

$$
\kappa_{i}=R_{j i} M_{j k}^{-1} R_{k i} \approx \alpha R_{j i}^{2} w_{j}
$$

where the map $w$ is an approximation pixel-diagonal of $M^{-1}$ built assuming white (uncorrelated) noise and $\alpha$ is a factor that compensates for the mean error we make by replacing $M^{-1}$ with $\vec{w}$. We determine $\alpha$ by evaluating a few pixels of the exact $\kappa$.

\subsubsection{Ad-hoc Filter}

Due to the time-domain noise model underestimating the amount of correlated noise in the data, we applied an extra adhoc filter to the maps. This is described in Appendix C, but has the effect of suppressing noise for scales $\gtrsim 0^{\circ}$. 1 .

\subsubsection{Point-source Handling}

During map-making, any samples that were within 0.8 of Venus, Mars, Jupiter, Saturn, Uranus, or Neptune were cut to avoid both the planets themselves and $0.1 \%-1 \%$-level contamination through the near sidelobes. In addition, any sample within $3^{\prime}$ of the bright asteroids Vesta, Pallas, Ceres, Iris, Eros, Hebe, Juno, Melpomene, Eunomia, Flora, Bamberga, Ganymed, Metis, Nausikaa, and Malasslia were cut.

To avoid false detections from variable point sources (e.g., blazars) we also cut point sources with a peak amplitude of at least $500 \mu \mathrm{K}$ out to the radius where the beam has damped them to $10 \mu \mathrm{K}$. For daytime data, the peak amplitude threshold was reduced to $150 \mu \mathrm{K}$ and the cut area was broadened by $\pm 1^{\prime}$ in azimuth and $-1^{\prime}$ to $4^{\prime}$ in elevation to account for the harderto-model daytime beam and pointing. A $500 \mu \mathrm{K}$ corresponds to about $49 / 37 / 23 \mathrm{mJy}$ in the f090/f150/f220 bands, and with this $2770 / 3054 / 1640$ point sources were cut in the night and $9252 / 7886 / 1713$ in the day. Point sources fainter than this (but still with $\mathrm{S} / \mathrm{N}>10$ ), of which there were $8868 / 5246 / 73$ for the night-time and $2382 / 413 / 0$ for daytime, were individually fit and subtracted from the time-ordered data.

\subsubsection{Dust Masking}

In theory all Galactic dust should be canceled by the mean sky subtraction, since this represents length scales too large to evolve over the course of our observations. However, in practice small time-variable errors in our detector calibration can make the dust appear to fluctuate slightly in brightness. For sufficiently bright regions of dust these fluctuations become big enough to induce a large number of false positives in the search. Ideally we would

\footnotetext{
45 This time save comes at a small cost. By using one $N^{-1}$ when building the numerator of Equation (10), but effectively a slightly different one in the denominator because of the approximation we have to do for $\kappa, N^{-1}$ no longer cancels in the expectation value and we introduce a small bias. This would have been avoided if we had computed the full $\hat{m}$ and then applied the same approximate $M^{-1}\left(U^{-1}\right)$ both in the numerator and denominator, but is ultimately corrected during debiasing (Section 4.6).
}

Table 2

Prior Parameter Ranges from Figure 15 of B19

\begin{tabular}{lccccc}
\hline \hline$M\left(M_{\oplus}\right)$ & $a(\mathrm{au})$ & $e$ & $q(\mathrm{au})$ & $Q(\mathrm{au})$ & $v_{\text {ref }}\left(\mathrm{yr}^{-1}\right)$ \\
\hline 5 & $350-450$ & $0.10-0.20$ & $280-405$ & $385-540$ & $1.58-1.82$ \\
5 & $450-550$ & $0.20-0.30$ & $315-440$ & $540-715$ & $1.75-1.99$ \\
10 & $650-750$ & $0.30-0.40$ & $390-525$ & $845-1050$ & $2.02-2.26$ \\
10 & $750-850$ & $0.40-0.50$ & $375-510$ & $1050-1275$ & $2.05-2.31$
\end{tabular}

Note. This is based on the best-fit parameter points for the $5 M_{\oplus}$ and $10 M_{\oplus}$ scenarios, to which uncertainty ranges of $a \pm 50$ au and $e \pm 0.05$ were added based on the resolution of the grid they used in their investigation. The planet mass, $M$, is given in Earth masses, $e$ is the eccentricity, $q$ is the perihelion distance, and $Q$ is the aphelion distance, both in au. Speed $v_{\text {ref }}$ is the planet's hypothetical speed at a reference location of $r_{\text {ref }}=500$ au. The planet's actual speed will be $v=v_{\text {ref }} \frac{r_{\text {ref }}^{2}}{r^{2}}$.

use the dust signal itself to calibrate the detectors in these regions, but for now we simply mask them.

We built a dust mask by high-pass filtering the Planck PR2 $545 \mathrm{GHz}$ map with the Butterworth filter $\beta(\ell, 1500,-5)$; (see Appendix $C$ ), selecting the $7 \%$ brightest pixels of the absolute value of the result, and growing the result by smoothing it with a Gaussian beam with $\sigma=7 ! 2$ and masking areas with value $>0.5$. This mask was applied to each $\rho, \kappa$ map. We found that the edges of the mask introduce some artifacts during the shiftand-stack search, so we additionally applied a $20^{\prime}$ larger mask before the final object detection step.

\subsection{Search Space}

The distance to and velocity of Planet 9 are relatively poorly determined, but we can infer rough limits on the acceptable fit from Figure 15 of B19, as shown in Table 2. We see that Planet 9's current distance is limited to $300 \mathrm{au} \lesssim r \lesssim 1300 \mathrm{au}$. Equation (1) for the heliocentric angular velocity of the planet can be re-expressed as

$$
\begin{aligned}
v & =v_{\text {ref }}\left(\frac{r}{500 \mathrm{au}}\right)^{-2} \\
v_{\text {ref }} & =1.93 \mathrm{yr}^{-1} \cdot \sqrt{a\left(1-e^{2}\right) /(500 \mathrm{au})}
\end{aligned}
$$

and from Table 2 we see that $v_{\text {ref }}$ is in the range 1.6 to $2 ! 3 \mathrm{yr}^{-1}$ for all of the acceptable fits. ${ }^{46}$ This means that only a hollow cone in our $r, v_{x}, v_{y}$ parameter space needs to be explored.

While in theory there is a continuum of possible parameter values inside this cone, in practice the limited angular resolution of the telescope means that very similar parameters are indistinguishable. From Equation (2) we see that getting the distance wrong by $\delta r$ results in a parallax ellipse that is bigger by

$$
\delta \theta_{r} \approx-0.14 \cdot \frac{\delta r}{10 \mathrm{au}}\left(\frac{500 \mathrm{au}}{r}\right)^{2} .
$$

Thus shift-stacking with the wrong distance leaves a residual ellipse with a radius of $\left|\delta \theta_{r}\right|$. If we step through distances in steps of $\Delta r$, then $\delta r$ will take on values in the range $\left[-\frac{\Delta r}{2}, \frac{\Delta r}{2}\right]$. Using Equations (16) and (A4) from Appendix A, we see that

\footnotetext{
46 The exact range depends on the assumptions we make for the acceptable range around each set of "best-fit" parameters Figure 15 of B19 gives, and the actual parameter search we performed was based the slightly different range 1 !'50< $v_{\text {ref }}<2$ !' 26 .
} 

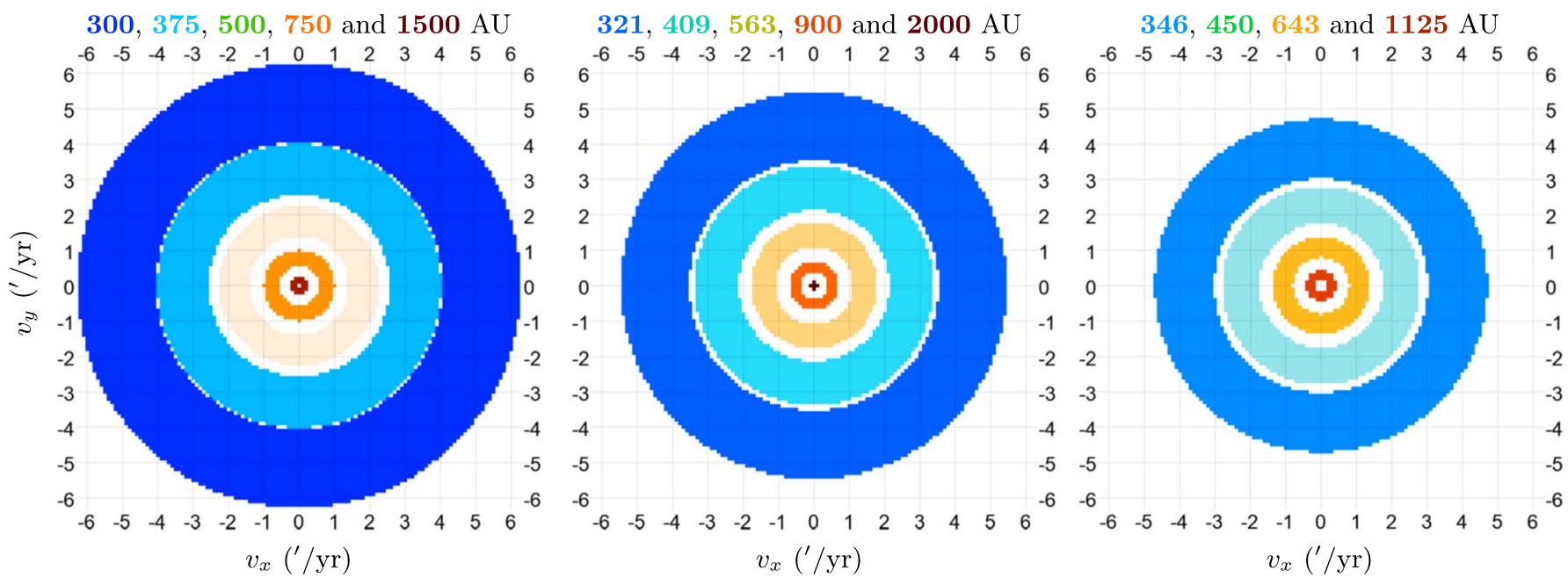

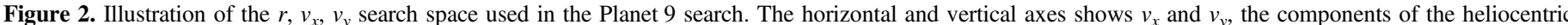

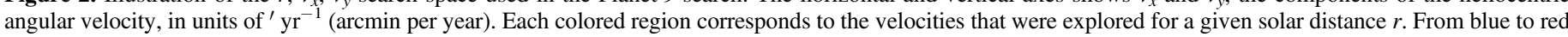

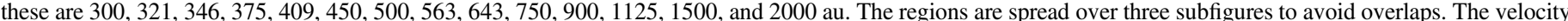
grid used in the search had a $0 ! 1 \mathrm{yr}^{-1}$ resolution, resulting in a total parameter volume of 25,837 cells.

A: Sun-centered raw signal

B: Earth-centered + mapmaker filter, 3-day obs

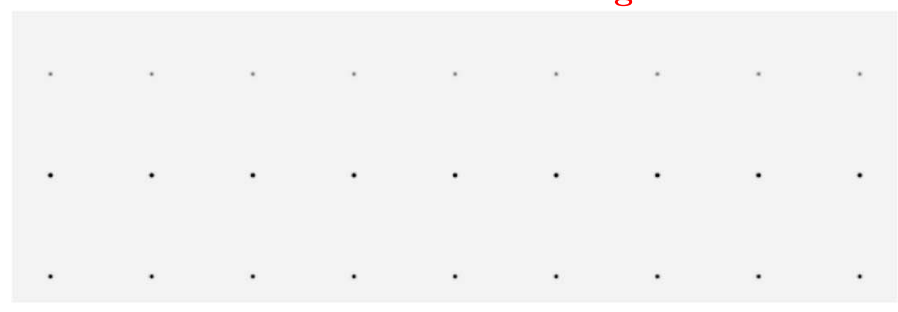

C: After injecting into noisy data

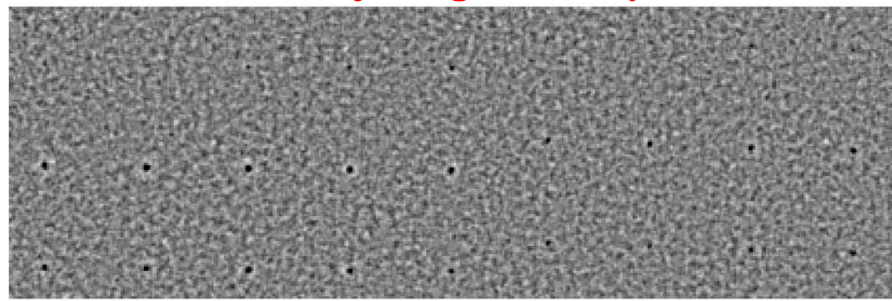

D: Plain mean of all observations

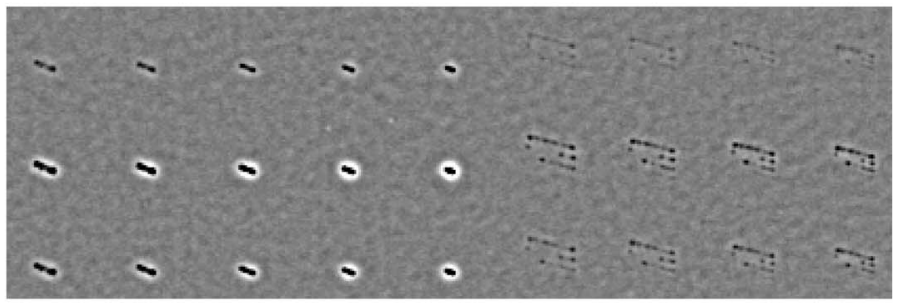

E: After shift +stack: Detection statistic $z$

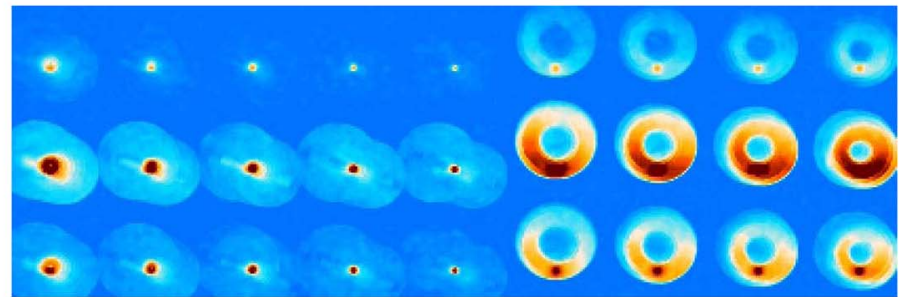

Figure 3. Example of injection and recovery of simulated objects used in Sections 4.6 and 4.7. A: objects of varying flux densities, distances, and velocities are simulated in a grid in Sun-centered coordinates. The simulated objects in this example are relatively bright, with f150 fluxes of 45,150 , and $100 \mathrm{mJy}$ from top to bottom and distances 750, 900, 1125, 1500, 2000, 300, 321, 346, and 375 au from left to right and correspondingly varying speeds. B: as in A, but in Earth-centered coordinates for an individual 3 day map. The displacement of the rows is due to the velocity and parallax. The faint rings are caused by the map-maker part of the matched filter. C: the result of injecting B into the real, noisy data, and applying the full matched filter. D: mean of all of the maps, without any shifting. The closest objects are greatly diluted by motion. The gaps in the smearing are caused by periods without observation. E: the shift-and-stack detection statistic ( $z$ ), showing a peak at the correct location of each object, surrounded by a lower-amplitude region corresponding to less optimal orbital solutions. This should be compared to the $z$ map column in Tables 3 and 4. 
Table 3

Top 10 Planet 9-Like Candidates, Sorted by the Detection Statistic z (see Section 4.7 or Appendix B for Definition)

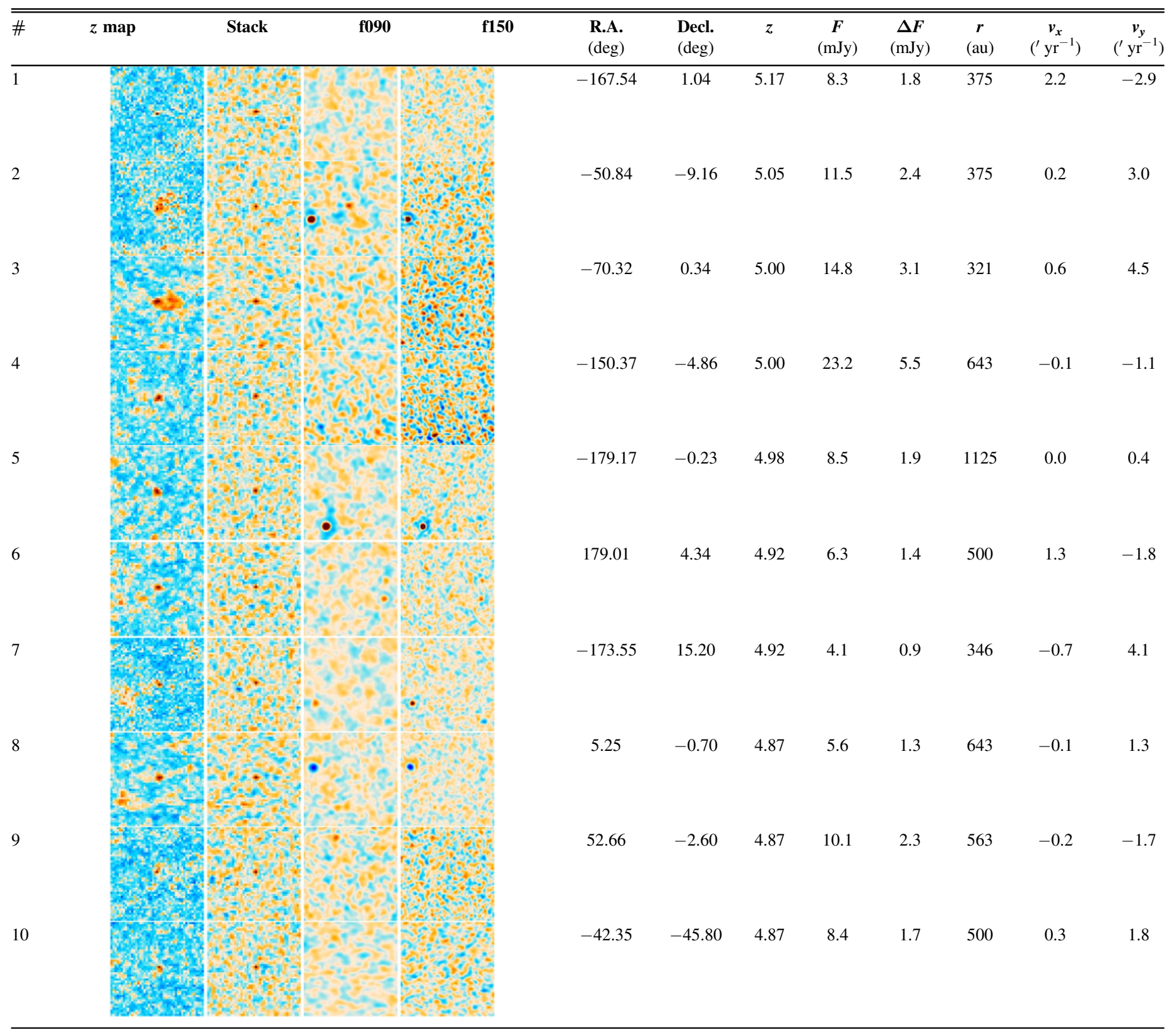

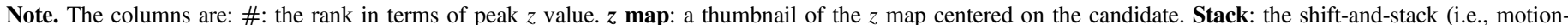

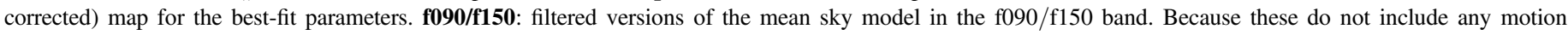

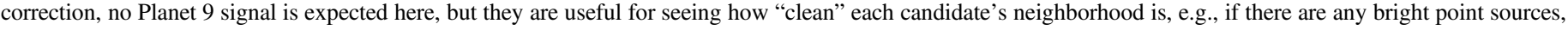

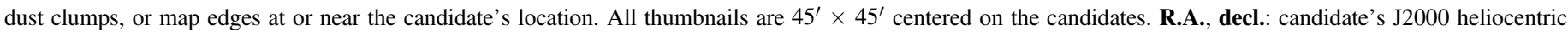

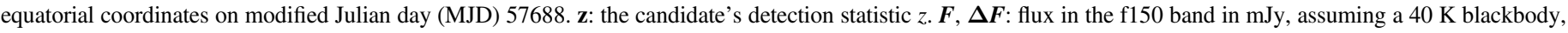
and its uncertainty. $\boldsymbol{r}$ : distance from the Sun, in au. $\boldsymbol{v}_{\boldsymbol{x}}, \boldsymbol{v}_{\boldsymbol{y}}$ : intrinsic motion in arcminutes per year.

on average, this increases the beam FWHM in quadrature by:

$$
\Delta \mathrm{FWHM}_{\mathrm{r}}=0.093 \cdot \frac{\Delta r}{10 \mathrm{au}}\left(\frac{500 \mathrm{au}}{r}\right)^{2} .
$$

Similarly, getting the speed wrong by $\delta v$ will over a time $T=t-t_{0}$ accumulate to a position error of

$$
\delta \theta_{v}=0 ! 3 \cdot \frac{\delta v}{0 ! 1 \mathrm{yr}^{-1}} \frac{T}{3 \mathrm{yr}} .
$$

For a velocity step of $\Delta v$ we get, using Equation (A6),

$$
\Delta \mathrm{FWHM}_{v}=0.29 \cdot \frac{\Delta v}{0 ! 1 \mathrm{yr}^{-1}} \frac{T}{3 \mathrm{yr}}
$$

where we have included a factor $\sqrt{2}$ in the numerical factor to take into account the smearing in both the $x$ and $y$ directions. The factor $T$ depends on when in the ACT observing campaign each observation was taken, but will at most be three years if we choose $t_{0}$ to be the midpoint of ACT observations. The integration time $\Delta t$ 
Table 4

Like Table 3, but for the General Candidates

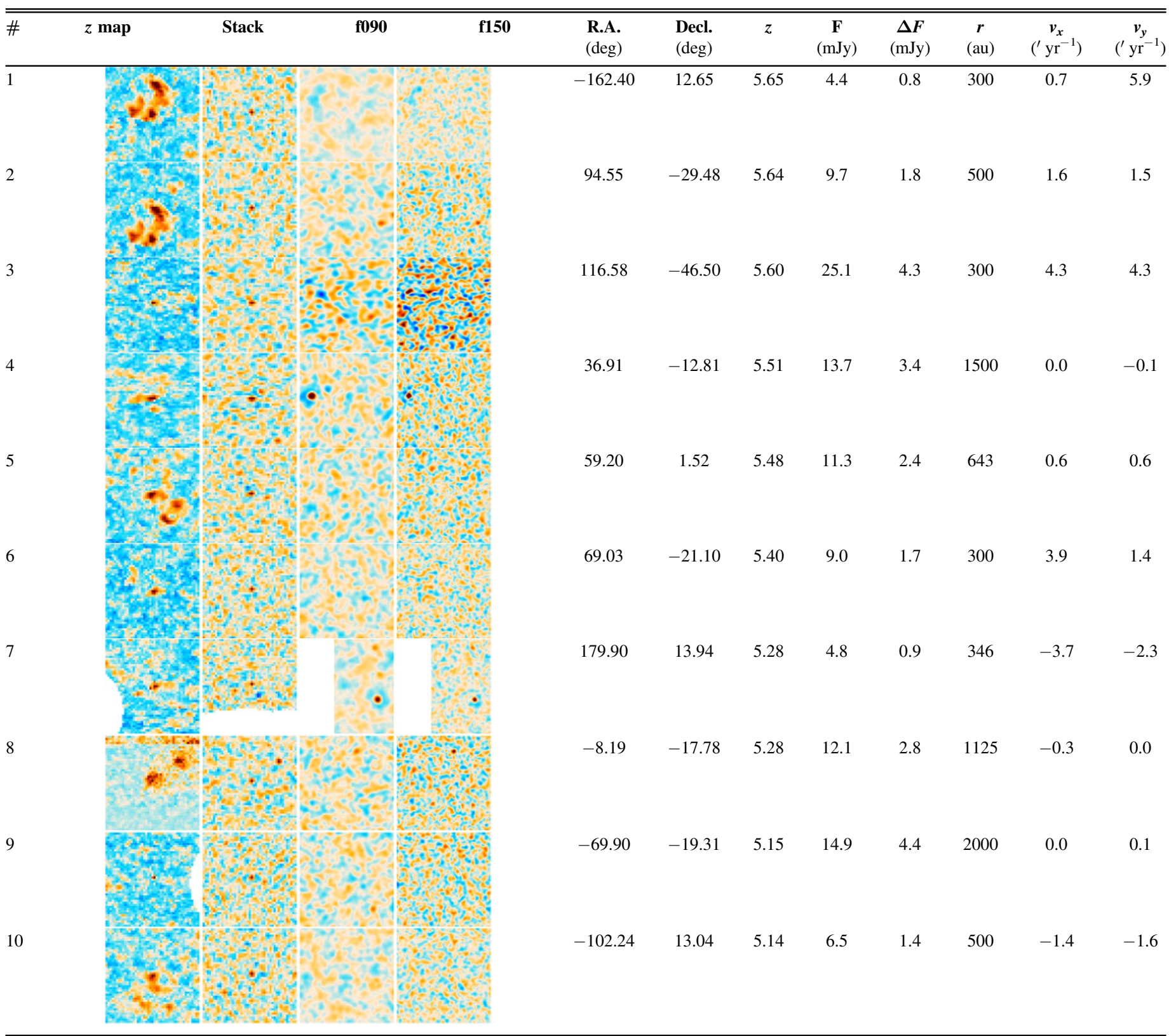

also results in smearing,

$$
\Delta \mathrm{FWHM}_{t}=0.080 \cdot \frac{500 \mathrm{au}}{r} \frac{\Delta t}{\text { day }}
$$

as does the pixel window

$$
\Delta \mathrm{FWHM}_{\text {pix }}=0.68 \cdot \frac{\text { res }}{1^{\prime}}
$$

where res is the pixel side length. ${ }^{47,}{ }^{48}$ Together these effects make up our smearing budget, and each must be chosen small enough that their combined effect does not overly degrade the $\mathrm{S} / \mathrm{N}$. We choose

\footnotetext{
47 This includes a factor of $\sqrt{2}$ because the pixels smear in two dimensions, but also a factor of $1 / \sqrt{2}$ because the noise also is being smoothed, counteracting some of the $\mathrm{S} / \mathrm{N}$ loss. This factor is only exactly $1 / \sqrt{2}$ when smoothing white noise with a Gaussian beam, but numerical tests show that it is an excellent approximation even for the top-hat smoothing effect of pixel binning.

48 In principle there is also some $\mathrm{S} / \mathrm{N}$ loss associated with the linear interpolation we use during shifting, but this is overwhelmed by the other effects.
}

1. $\Delta v=0.1 \mathrm{yr} \Rightarrow \Delta \mathrm{FWHM}_{v}=0.29$ for $T=3 \mathrm{yr}$.

2. $\Delta t=3$ days $\Rightarrow \Delta \mathrm{FWHM}_{t}=0.40$ for $r=300 \mathrm{au}$, which is the closest distance we will consider.

3. $\Delta r=33 \mathrm{au} \cdot\left(\frac{500 \mathrm{au}}{r}\right) \Rightarrow \Delta \mathrm{FWHM}_{r}=0$ ' 31 . This results in the discrete set of distances: $300,321,346,375,409$, 450, 500, 563, 643, 750, 900, 1125, 1500, and 2000 au. The last two distance bins are more distant than Planet 9 is likely to be, but are included because of their low computational cost.

4. res $=1^{\prime} \Rightarrow \Delta \mathrm{FWHM}_{\mathrm{pix}}=0$ '.68. That is, we use a pixel size of $1^{\prime} .49$

\footnotetext{
49 In practice raw maps were built at 0.5 resolution and were only downsampled (by averaging blocks of $2 \times 2$ pixels) to $1^{\prime}$ resolution in the matched filter (that is, the $\rho$ and $\kappa$ maps were downsampled). Working with a higher resolution until this point reduces the aliasing one would otherwise get from working with pixels of comparable size to the FWHM.
} 

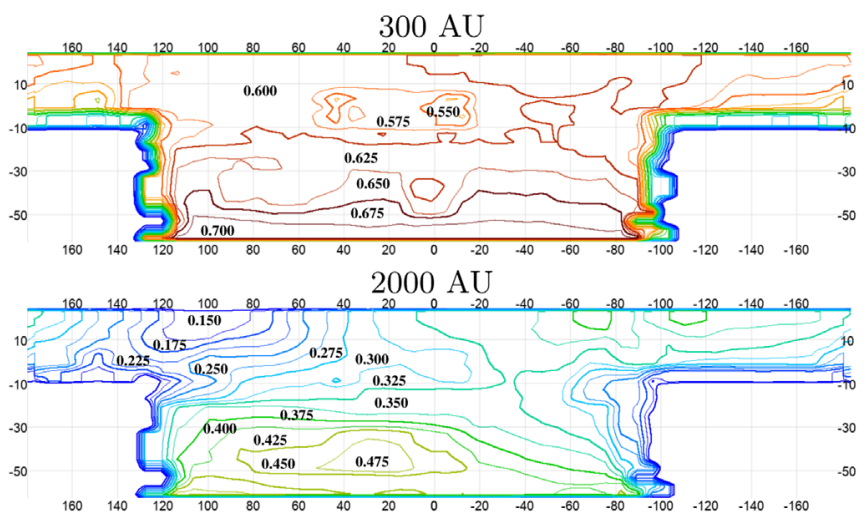

Figure 4. Contour plot of the bias factor recovered from the simulations described in Section 4.6. This is defined as the fraction of the true flux density that is recovered. The source of the bias is the mean sky subtraction described in Section 4.3.4. The top panel shows the bias for sources at $300 \mathrm{au}$; the bottom at $2000 \mathrm{au}$. The color scale goes from 0 (blue; all flux density lost) to 0.70 (red, $70 \%$ of flux density recovered), with a contour interval of 0.025 . The horizontal and vertical axes are R.A. and decl., respectively. We divide by these factors to debias the recovered flux densities.

These combine in quadrature to $\triangle \mathrm{FWHM}=0.90$, which when combined with our beams represents a $9 / 19 / 34 \%$ increase in beam size and loss in $\mathrm{S} / \mathrm{N}$ in the f090/f150/f220 bands respectively. The largest contribution to this is the $1^{\prime}$ pixel size. With a 0.5 pixel size these numbers would instead have been $5 / 11 / 21 \%$ at a cost of $4 \times$ as high $\mathrm{CPU}$ and memory budgets. We might consider using smaller pixels when we revisit this in the future.

The full, quantized search space is visualized in Figure 2. In total the $r, v_{x}, v_{y}$ parameter space has 25,837 cells.

\subsection{Shift-and-stack Implementation}

After splitting the 2013-2019 ACT data set into 3 day chunks and building matched filter maps for each, we were left with 3834 pairs of $\rho$ and $\kappa$ maps taking up a total of 1.9 TB of disk space. Since these maps are in units of equivalent flux density at the reference frequency $\nu_{0}=150 \mathrm{GHz}$, maps from different arrays and bandpasses that were observed at the same time, and hence all have the same shifts, can be directly combined before the main shiftand-stack search. This resulted in a more manageable 787 pairs taking up $220 \mathrm{~GB}$.

The analysis was performed in $10^{\circ} \times 10^{\circ}$ tiles with an additional $1^{\circ}$ padding on all sides using data "belonging" to neighboring tiles to avoid discontinuities at tile edges. For each tile we loop over our parameter space and keep track of the highest-S/N value of $v_{x}$ and $v_{y}$ in each pixel for each value of $r$. This is illustrated in the pseudo-code below:

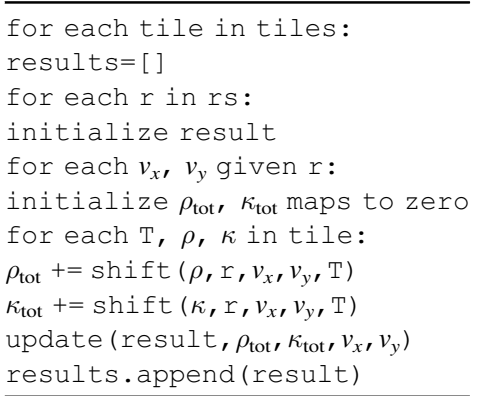

Here, $r$ takes on the values $300,321,346,375,409,450,500$, $563,643,750,900,1125,1500$, and 2000 au. For each value we visit all velocities $v_{x}=i \Delta v, v_{y}=j \Delta v$, where $i$ and $j$ are integers and

$$
\begin{aligned}
& 1 ! 6 \mathrm{yr}^{-1} \cdot\left(\frac{r}{500 \mathrm{au}}\right)^{-2}-\Delta v \leqslant \sqrt{v_{x}^{2}+v_{y}^{2}} \\
& \leqslant 2 ! 3 \mathrm{yr}^{-1} \cdot\left(\frac{r}{500 \mathrm{au}}\right)^{-2} .
\end{aligned}
$$

The function shift applies the coordinate transformation from observed coordinates at time $t=t_{0}+T$ to heliocentric coordinates at time $t_{0}$, taking into account both parallax for the distance $r$ and the planet's angular velocity $v_{x}, v_{y}$. We use bilinear interpolation to allow for fractional pixel shifts. This function is the most time-critical part of the search, so it was implemented in optimized C using AVX intrinsics and OpenMP parallelization. Since the distance and direction each pixel is displaced changes slowly as a function of position in the map, we use the same displacement for blocks of $8 \times 8$ pixels, saving a large number of trigonometric operations at no loss of $\mathrm{S} / \mathrm{N}$. Overall our implementation is 480 times faster than a straightforward numpy/scipy implementation.

The function update updates result to maintain a running record of the highest $\mathrm{S} / \mathrm{N}$ observed in each pixel, and what value of $\rho_{\text {tot }}, \kappa_{\text {tot }}, v_{x}$, and $v_{y}$ that occurred for. We maintain one such result for each value of $r$ because both bias from mean sky subtraction and the appropriate $\mathrm{S} / \mathrm{N}$ threshold for a detection (which depends on the effective number of trials) depend on $r$.

\subsection{Simulations and Debiasing}

Mean sky subtraction mainly removes the static parts of the sky, but it also subtracts some of the signal from moving objects. These appear as a smeared-out tracks in the mean sky map, and since part of an object's track necessarily overlaps with its position in each individual exposure, mean sky subtraction will always lead to a loss of signal power. The size of the bias is both distance dependent (because more distant objects move less and hence overlap more with the mean sky) and position dependent (because areas with less coverage will see less of the object's motion).

To characterize this bias we performed a set of signal injection and recovery simulations. We created a catalog of fake planets with the same flux density forming an equispaced grid in heliocentric R.A. and decl. at $t=t_{0}$, with values of $r$ stepping through the 14 values we consider in the parameter search for every 14 grid positions in R.A., and $v$ taking on the corresponding 14 values 1 ! $80,1.57,1 ! 35,1$ ! $15,0 ! 97,0 ! 80,0$. $65,0.51,0.39$, $0 ! 29,0 ! 20,0 ! 13,0 \prime 07$, and $0.04 \mathrm{yr}^{-1}$. An example of such a grid of simulated objects is shown in panel A of Figure 3.

Given this catalog, we compute the Earth-centered position of each object for each 3 day map, and use the instrument beam, bandcenter, and map-maker filter to simulate a signalonly image $m_{\text {sim }}$ of these sources in the same units as our data maps: $\mathrm{CMB}$ temperature increment in $\mu \mathrm{K}$. This is shown in panel $\mathrm{B}$ of the figure.

These were used to build new matched filter maps $\rho_{\text {sim }}^{\text {raw }}=R^{T} w o m_{\text {sim }}$, where $\circ$ is the element-wise product and $w$ is the white noise inverse variance map from Section 4.3.4. ${ }^{50}$ Using $w o m_{\text {sim }}$ instead of Equation (13) is an approximation, but

\footnotetext{
50 Indices for the individual time chunks and bandpasses have been suppressed here for readability.
} 


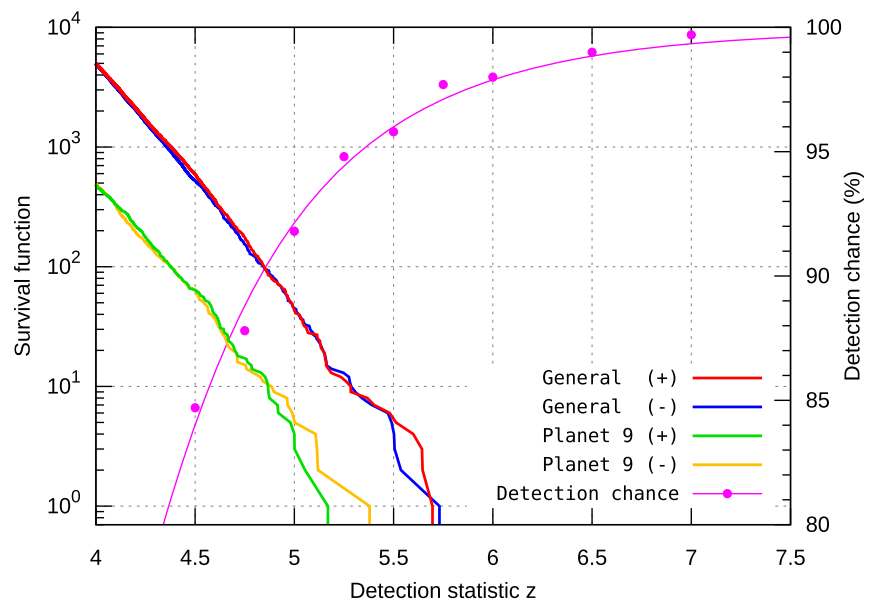

Figure 5. Left axis: distribution of candidate detections for the Planet 9-like (green) and general (red) search compared to sign-flipped versions of the same searches (blue and yellow, respectively), as a function of the detection statistic $z$. No signal is expected in the sign-flipped search, but it shares the same noise properties and many of the systematics (e.g., variable point sources and edge artifacts), so it gives a good estimate of the background detection rate. The lack of excess events in the positive curves vs. the negative ones (beyond the scatter expected from Poisson sample variance) means that we do not have any significant detections. Right axis: The probability of recovering an injected object as a function of $z$ (magenta). The detection probability is $95 \%$ by $z=5.3$.

based on a small number of full time-domain simulations it appears to be accurate to $<5 \%$.

To capture the effect of mean sky subtraction we define the mean flux density map for the simulations

$$
F_{\text {mean }}=\rho_{\text {sim,tot }} / \kappa_{\text {tot }}, \rho_{\text {sim,tot }}=\sum_{i} \rho_{\text {sim }, i}^{\text {raw }}
$$

where $i$ loops over all of the individual maps and the division is element-wise. Panels C and D of Figure 3 shows examples of individual and mean matched filter maps, but differ from the ones described here by not being noise free (see Section 4.9). Using these maps we define the mean sky subtracted simulations

$$
\rho_{\text {sim }}=\rho_{\text {sim }}^{\text {raw }}-\kappa F_{\text {mean }},
$$

This was done individually for each bandpass, both to avoid mixing maps with different beams and to reflect what was done to the actual data.

Finally, we ran the shift-and-stack procedure from Section 4.5 on the simulated data set, and read off the recovered flux density for each simulated source. We find practically no dependence on the direction of the velocity, and therefore average the data points for different velocities for our final bias model, resulting in bias maps $\boldsymbol{b}(r)$ with a resolution of $7^{\circ} \times 4^{\circ}$. These are shown for the closest and furthest Planet 9 distance considered in Figure 4. The bias changes smoothly with position and is well resolved even with these large pixels. The standard deviation of the data points going into each pixel is about $0.5 \%$, which we take as the uncertainty on our bias maps. We use this to define $\rho_{\text {tot }}^{\text {debiased }}=\rho_{\text {tot }}$ ob and $\kappa_{\text {tot }}^{\text {debiased }}=\kappa_{\text {tot }} \circ \boldsymbol{b}^{2}$, from which bias-free flux densities can be recovered via Equation (10).

\subsection{Significance}

Our search method results in a map for each $r$ where each pixel has the maximum $\mathrm{S} / \mathrm{N}$ across all of the velocity parameters for that $r$. To construct a list of detection candidates and detection limit maps we need to know the background distribution of these $\mathrm{S} / \mathrm{N}$ values. This is made difficult by the varying depth, and varying temporal and spatial distribution of the data used in the search. The effective number of trials is a strong function of $r$, and the individual trials are correlated, with the correlation depending on how densely the ACT observations covers each spot of the map. The $\mathrm{S} / \mathrm{N}$ distribution should therefore vary both as a function of $r$ and position.

The simple approach of multiplying the number of beams in the map ( $\sim 30$ million) with the total trial number $(25,837)$ to get a total number of trials $\left(\sim 10^{12}\right)$ and a corresponding Gaussian quantile $(7 \sigma)$ does not work. Aside from overestimating the effective number of trials, it would also lead to the search grossly preferring candidates with low $r$ by not penalizing the much larger parameter space for low $r$ compared to high $r$.

Instead we will take the approach of transforming $\mathrm{S} / \mathrm{N}$ into an overall detection statistic $z$ that follows a simple, uniform Gaussian distribution, at least for its high- $z$ tail. This procedure is described in Appendix B, where we find that

$$
z=\xi(\mathrm{S} / \mathrm{N})=\left(\mathrm{S} / \mathrm{N}-\mu_{z}\right) / \sigma_{z}
$$

where $\mu_{z}$ and $\sigma_{z}$ are functions of distance $r$ and position in the map.

\subsection{Candidate Identification}

With the normalized detection statistic $z$ in hand, we build a set of preliminary candidate detections by selecting peaks with $z>3.5$. Given the large sky area covered, this low threshold will result in a large number of candidates, the vast majority of which would of course simply be noise fluctuations (especially considering that we expect at most one real object), but that allows us to get a good handle on the background distribution that any real objects would stand out from.

To better understand the background, we took advantage of the fact that the planet signature would be positive in our maps and repeated the whole search with the sign of all the data flipped. No signal is expected in the sign-flipped search, but it shares the same noise properties and many of the systematics (e.g., variable point sources and edge artifacts), so it gives a good estimate of the background detection rate.

We classified each candidate as Planet 9-like or general based on whether they satisfied the expected bounds on Planet9's orbital inclination, $10^{\circ}<i<30^{\circ}$ (B19). ${ }^{51}$ The inclination is not one of the free parameters of our fit, but we can approximate this selection by transforming the candidate coordinates and velocities into ecliptic coordinates, and requiring

$$
10^{\circ}<\hat{i}<30^{\circ}, \hat{i}=\sqrt{\beta^{2}+v_{\beta}^{2} / v_{\lambda}^{2}}
$$

and where $\lambda, \beta$ are the ecliptic longitude and latitude, respectively, and $v_{\lambda}, v_{\beta}$ are the velocity components in those directions. ${ }^{52}$ The

\footnotetext{
51 Note that this inclination bound is the only difference between the "Planet 9-like" and "general" categories. Because both of them are based on a parameter search that only considered distances and velocities reasonable for Planet 9 (see Section 4.4), even the "general" search is not sensitive to planets with extreme ellipticity or $r<300$ au.

52 In practice we accidentally used $v_{\beta}{ }^{\prime}=\max \left(\left|v_{\beta}\right|-\Delta v, 0\right)$ and $v_{\lambda}{ }^{\prime}=$ $\max \left(\left|v_{\lambda}\right|-\Delta v, 0\right)$ instead. These were supposed to avoid division by zero, but by using the wrong sign in front of $\Delta v$ they instead increased the likelihood for this. In practice this has negligible effects on our results, since only the highest distance bin $r=2000$ au has low enough speeds that a $0.05^{\prime} \mathrm{yr}^{-1}$ difference would matter.
} 


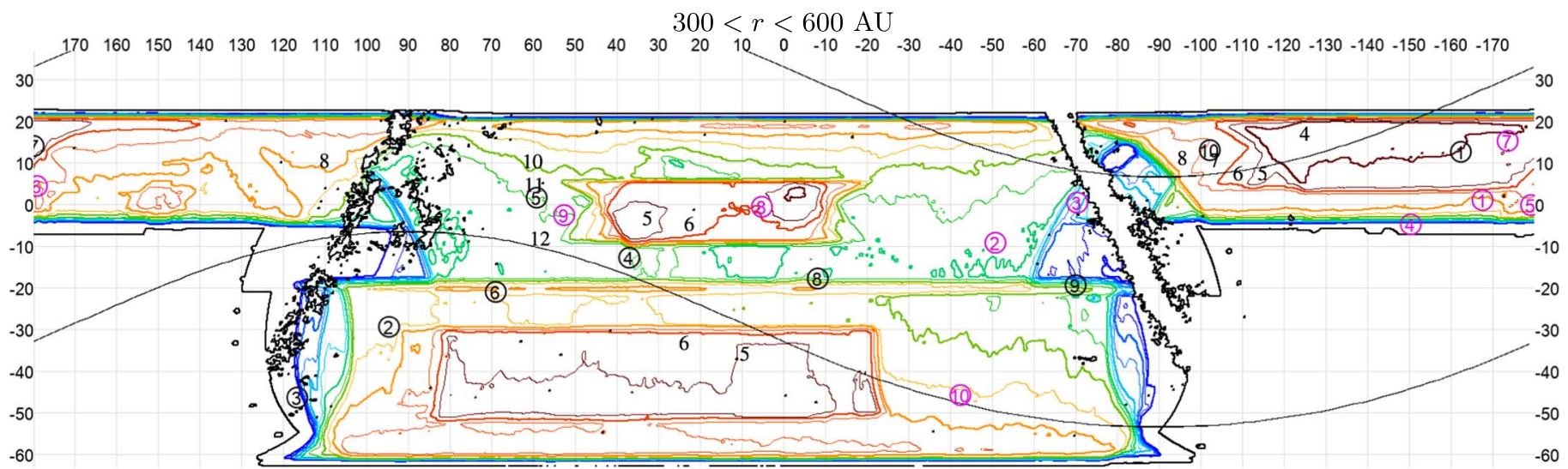

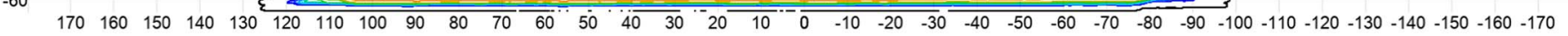
$r=1500 \mathrm{AU}$

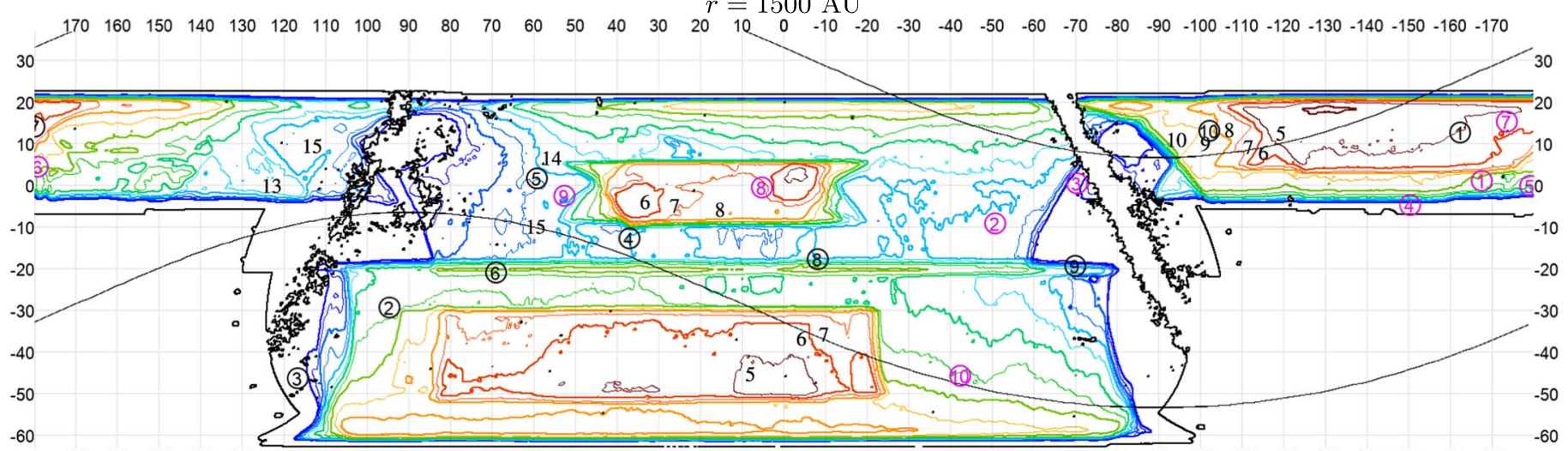

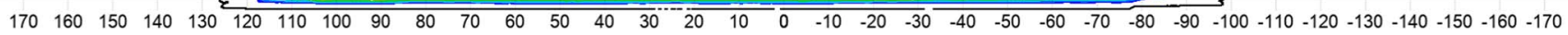

Figure 6. Flux limit for the general Planet 9 search for distance $300<r<600$ au (top) and $r=2000$ au (bottom) in equatorial coordinates. Objects brighter than this would be part of our top 10 candidate list. The contours go from red $(4 \mathrm{mJy})$ to blue $(20 \mathrm{mJy})$ in steps of $1 \mathrm{mJy}$. Some individual contour lines are labeled (plain black numbers) with their corresponding depth, for convenience. The flux density limit depends on the size of the parameter search space, which shrinks with $r$ leading to a lower flux density limit; and the loss from mean sky subtraction, which raises the flux density limit. These effects mostly balance each other for $300<r<600$, while the mean sky subtraction loss dominates at higher $r$, leading to a gradually increasing flux density limit. The circled numbers show the locations of the top 10 candidates from the general (black) and Planet 9-like (magenta) searches. The thin black curves delimit the area with inclination less than $30^{\circ}$. The point-source mask was left out from this plot, but its effect can be seen in Figure 7.

formula for $\hat{i}$ assumes that orbits have $\beta(\lambda)=i \sin \left(\lambda-\lambda_{0}\right)$, which is a decent approximation as long as $i$ is small.

Finally, the top 100 candidates from each list were visually inspected using the diagnostic maps below, and candidates with obvious problems were cut. ${ }^{53}$

1. Maps of the detection statistic $z$, to ensure that there is a sensible local maximum at the candidate location. The edges of the mask sometimes cause extended artifacts that are easily identified this way.

2. The shift-stacked maps using the best-fit orbital parameters for each candidate, again to ensure a compact, isolated maximum.

3. Raw sky maps for the f090 and $\mathrm{f} 150$ bands at the candidate location, to ensure that there is not an unmasked point source or dust structure there.

4. Individual 3 day matched filter maps, which were useful for disqualifying false positives caused by variable point sources and transients.

With the exception of the 3 day maps, these are shown as the image columns in Tables 3 and 4.

\footnotetext{
${ }^{53}$ Below the top 100 the statistics are completely dominated by noise fluctuations, and any artifacts would be hard to distinguish from noise anyway because of the low $\mathrm{S} / \mathrm{N}$.
}

\subsection{Flux and Distance Limits}

It is useful to be able to translate the survey depth into detection limit maps. To do this, we need the false negative rate as a function of the detection statistic $z$. We found this by repeating the signal injection, search, and detection procedure from Section 4.6 with two important differences:

1. Simulated sources were added to the data instead of replacing it, resulting in noisy simulations.

2. For each simulated source we chose a target $z \in\{4.50$, $4.75,5.00,5.25,5.50,5.75,6.00,6.50,7.00,8.00,10.00$, $15.00\}$, translated this into an $\mathrm{S} / \mathrm{N}$ ratio using $\xi^{-1}(z)$; (see Section 4.7 and Appendix B), and combined it with the local survey depth to define a simulated flux density $s=\xi^{-1}(z) / \sqrt{\kappa_{\text {tot }}^{\text {debiased }}}$.

We then ran the standard mean sky subtraction and candidate search on the maps, and computed the fraction of the injected sources that were ultimately recovered as a function of $z$. The result is plotted as the curve "detection chance" in Figure 5. Overall we find that a source bright enough to correspond to $z=5.3$ has a $95 \%$ chance of being detected. Hence, the $95 \%$ flux density detection limit map is given by

$$
s_{\lim }^{95 \%}=\xi^{-1}(5.3) / \sqrt{\kappa_{\text {tot }}^{\text {debiased }}} \text {. }
$$




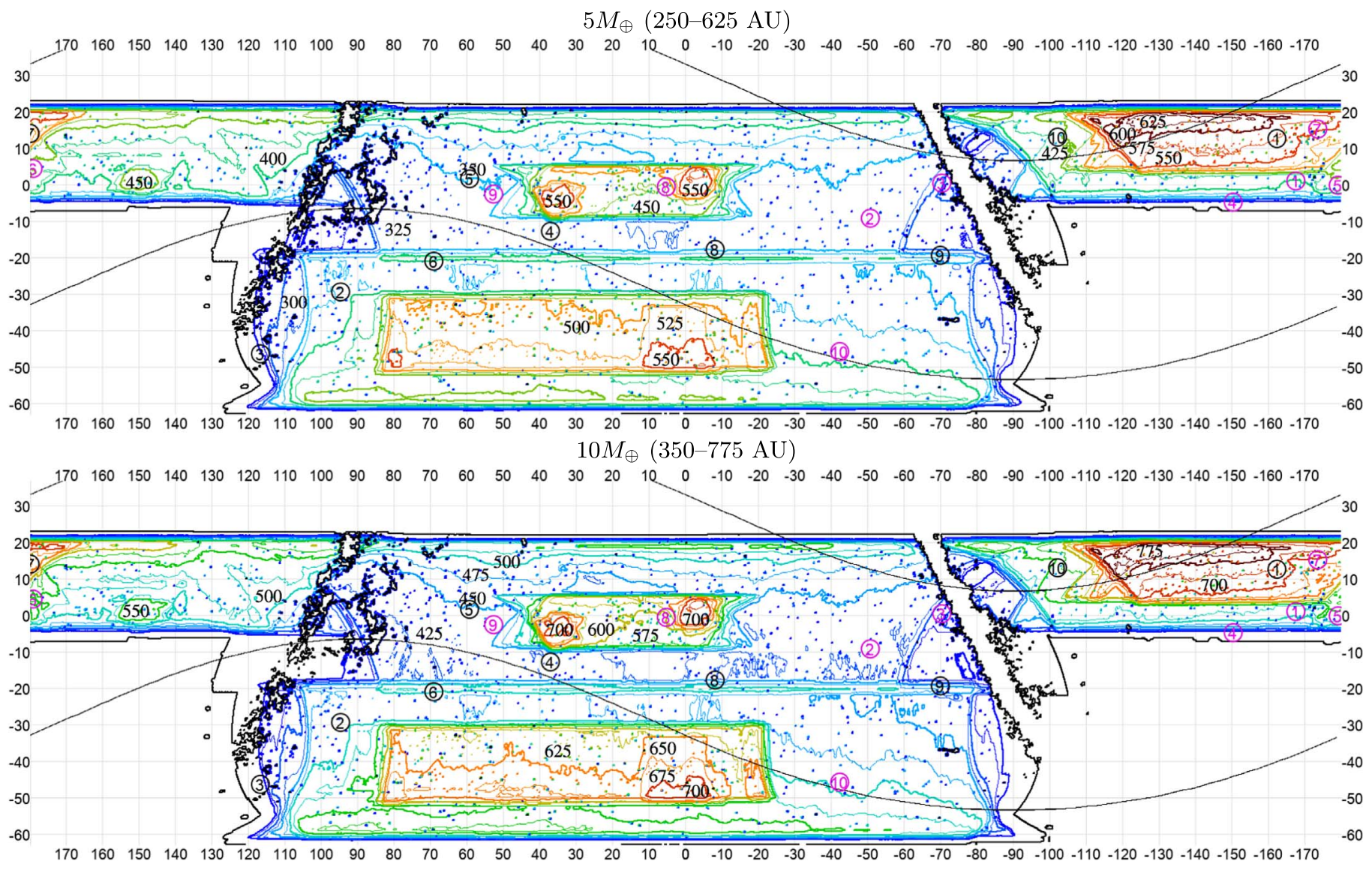

Figure 7. Top: distance limit for detection of a $5 M_{\oplus}$ Planet 9 with the nominal composition from Section 2 . Contours go from 250 au (blue) to 550 au (red) in steps of $25 \mathrm{au}$, with some contours labeled for convenience. Note that $r<300$ au were not included in the search, so areas with a distance limit of $\lesssim 300$ au do not meaningfully constrain Planet 9. The circled numbers show the locations of the top 10 candidates from the general (black) and Planet 9-like (magenta) searches. The little colored dots are caused by the point-source mask. Its effect appears exaggerated because of the low resolution of the plot-in reality this mask only affects a tiny fraction of the sky. Bottom: as above, but for a $10 M_{\oplus}$ Planet 9 . The contours here go from 350 au (blue) to 750 au (red).

Aside from its position dependence this limit is also distance dependent, since both $\xi$ and $\kappa_{\text {tot }}^{\text {debiased }}$ depend on $r$.

Given a model for Planet 9's luminosity we can translate the flux density limit to a distance limit. Since the flux density falls with the square of the distance, the distance limit $r_{\mathrm{lim}}^{95 \%}$ can be found as the solution to the equation

$$
\left(r_{\text {ref }} / r\right)^{2} s_{\text {ref }}=s_{\text {lim }}^{95 \%}\left(r_{\text {lim }}\right)
$$

with Table 1 showing examples of the reference flux $s_{\text {ref }}$ for $r_{\text {ref }}=500$ au for different Planet 9 scenarios. ${ }^{54}$

\section{Results}

The search resulted in 38,000 raw candidates, of which 3500 and 35,000 fell into the Planet 9-like and general categories, respectively. Manual inspection of the top 100 candidates led to 3 Planet 9-like and 17 general candidates being cut. These included the first three transients detected by ACT, which were published in a separate paper (Naess et al. 2021). The top ten candidates from the Planet 9-like and general searches are shown in Tables 3 and 4. The full candidate distribution is shown in Figure 5 and is identical to within sample variance for both the normal and sign-inverted searches. The lack of excess

\footnotetext{
54 We assume that $s_{\text {lim }}^{95 \%}$ changes linearly between the discrete set of distances $r \in\{300,321,346,375,409,450,500,563,643,750,900,1125,1500,2000\}$ au where we computed it.
}

events in the distribution of normal candidates versus signinverted candidates means that we have no statistically significant detections.

Given our nondetection, we constrain the flux density from Planet 9 or similar objects in the outer solar system to be $<4-12 \mathrm{mJy}$ (95\% confidence) for $r \geqslant 300$ au inside our survey area, depending on local survey depth. This limit is approximately distance independent in the range 300 au $\leqslant$ $r \leqslant 600$ au, after which it gradually worsens to $<5-15$ mJy by 1500 au. We show a map of the flux density limit in Figure 6, along with the locations of the top 10 candidates from the Planet 9-like and general searches.

Figure 7 shows the corresponding distance limits for the nominal $5 M_{\oplus}$ and $10 M_{\oplus}$ scenarios from Section 2. In the shallower parts of our survey area, a $5 M_{\oplus}$ Planet 9 would need to be more distant than 325 au to evade detection. This increases to 625 au in the deepest parts of our survey. For a $10 M_{\oplus}$ planet these numbers increase to 425 au and 775 au, respectively.

We cover quite low Galactic latitudes, but parts of the Galaxy are still masked. This is usually confined to $|b|<2^{\circ} .5$, but it is not uncommon for the mask to extend beyond this to cover features like the Orion Nebula.

\section{Discussion}

As the possible signal curves in Figure 1 showed, ACT's nondetection is not surprising, especially considering that a 

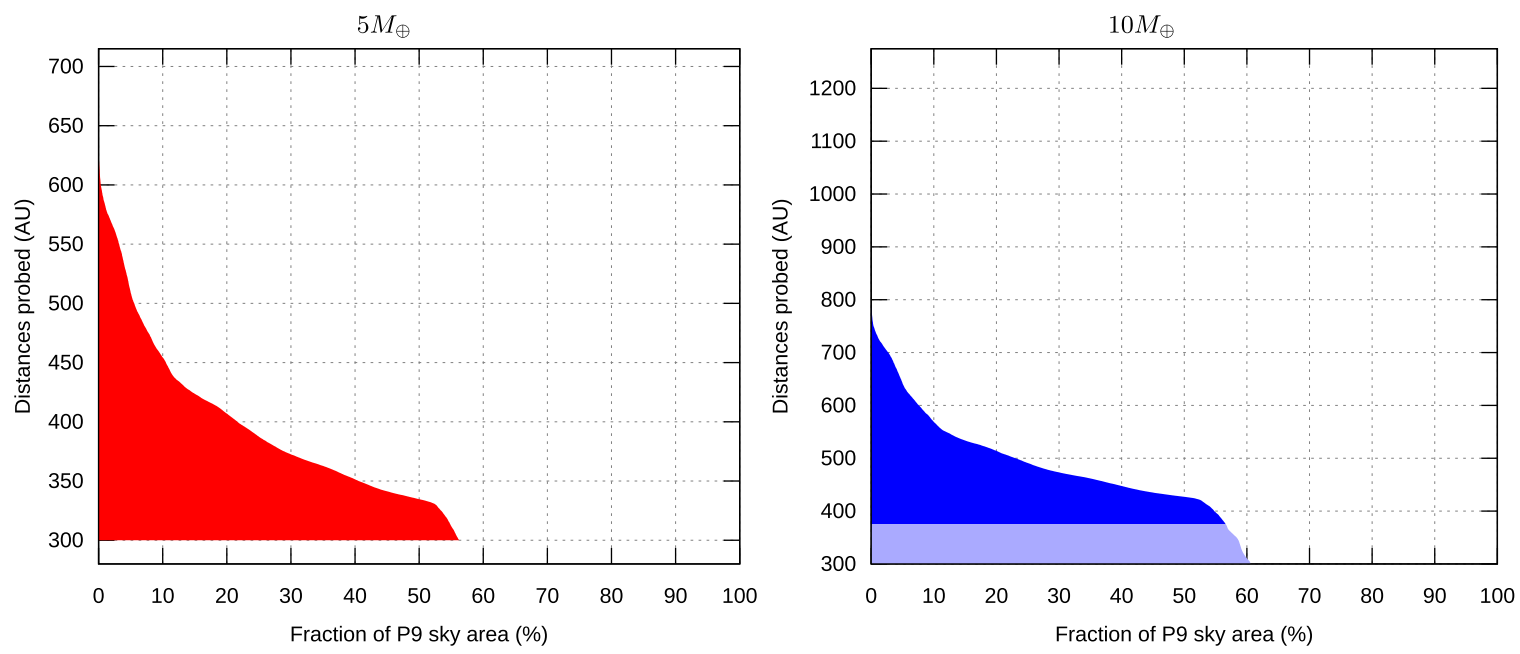

Figure 8. Our Planet 9 exclusion limit distribution with the nominal composition from Section 2, showing the distance range we can exclude over a given fraction of the Planet 9 expected sky area $\left(|\beta|<30^{\circ}\right)$. The value on the $x$-axis gives the fraction of the Planet 9 sky area where our bounds are at least as good as the distance given on the $y$-axis. This is what one gets if one sorts the values in Figure 7 in descending order. Left: the nominal $5 M_{\oplus}$ Planet 9 is predicted to be between roughly 280 and 715 au distant (see Table 2). This curve shows that we are sensitive up to 600 au over a few percent of the Planet 9 sky area; up to 450 au over $10 \%$ of that area; 300 au to at least 350 au over $40 \%$ of the area; and so on. The colored fraction of the graph gives a rough idea of what fraction of the Planet 9 parameter space we probe. Right: as left, but for the $10 M_{\oplus}$ version of the planet. The light shaded region represents distances we probe that are closer than what is allowed by the prior.

planet in an eccentric orbit moves more slowly near aphelion, and is therefore more likely to be located there. Planet 9's aphelion is predicted to be around R.A. $\approx 60^{\circ}$, an area where the ACT coverage is quite shallow, corresponding to a $5 M_{\oplus}$ detection limit of about 350 au. For comparison, the smallest expected aphelion distance is a bit less than 400 au (Table 2). Hence, at its current depth, ACT cannot expect to see Planet 9 if it is near aphelion.

Because B19 does not provide a well-defined prior volume, it is hard to quantify what fraction of the Planet 9 parameter space we have probed, but we can make a few simple estimates. Figure 8 shows the distribution of our distance limits for the Planet 9 relevant parts of the sky $\left(|i|<30^{\circ}\right)$, and compares them to the $\sim 300-700$ au and $\sim 400-1300$ au allowed distance range for a $5 M_{\oplus}$ and $10 M_{\oplus}$ Planet 9 , respectively. We probe about $13 \%$ and $8 \%$ of this distance-position space. However, that does not take into account the fact that the furthest Planet 9 distances are only expected to occur in some parts of the sky. The spatial dependence of the predicted Planet 9 distance range is shown in Figure 9, and taking it into account, our numbers improve to $17 \%$ and $9 \%$, respectively.

We can also compare our bounds to the general parameter space for undiscovered solar system objects. This is done in Figure 10, though with some large caveats, since we made several assumptions specific to Planet 9 in our search.

Our bounds can also be compared to recent bounds on Planet 9 based on its gravitational influence on the orbit of Jupiter and Saturn, which are known to high precision due to data from the Juno and Cassini probes. Fienga et al. (2020) use the INPOP19a planetary ephemerides to put strong bounds on Planet 9 , excluding a $5 M_{\oplus}$ version closer than about 550 au and a $10 M_{\oplus}$ version closer than about $700 \mathrm{au}$, and allowing it only in limited regions of the sky beyond that. Their preferred regions happen to nearly coincide with our deepest areas, but despite that our bounds only approach theirs, and do not exceed them.

The upcoming Simons Observatory (SO; SO Collaboration 2019) will substantially improve on these bounds. Extrapolating our current results to the expected depth of the combined
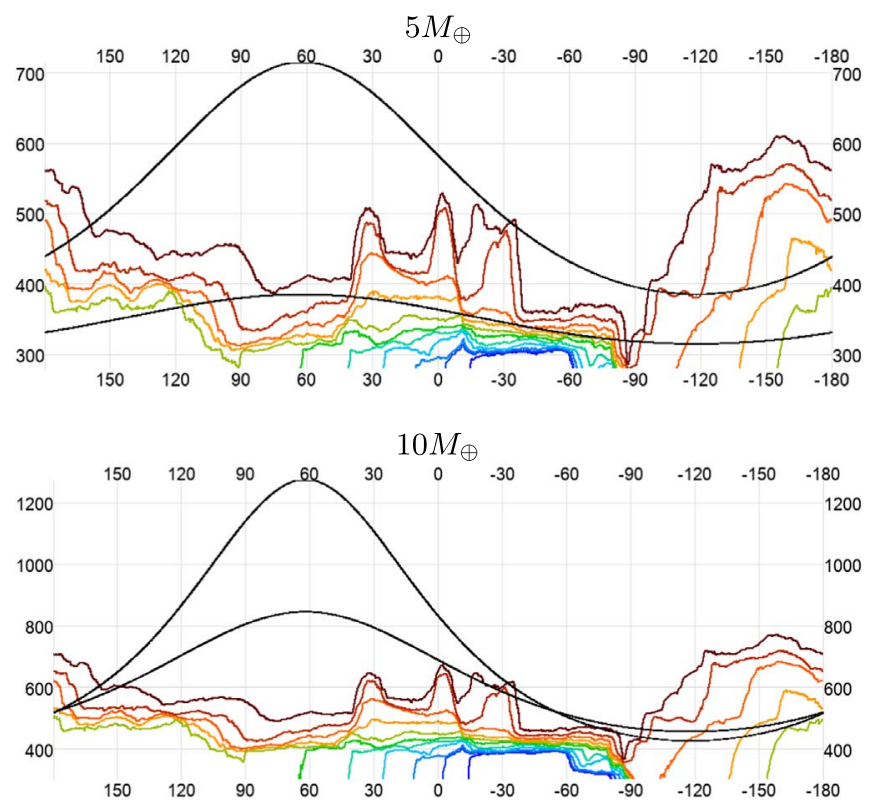

Figure 9. Distance limit distribution as a function of ecliptic longitude compared to the Planet 9 orbit. The $x$-axis is ecliptic longitude in degrees. The $y$-axis is the planet's current distance from the Sun. The colored curves are deciles of the distance limit distribution for the Planet 9-like search, from blue $(0 \%)$ to red $(100 \%)$. The bottom and top black curves represent, respectively, a low- $a$, low- $e$ case and a high- $a$, high- $e$ case. These roughly bracket the Planet 9 prior space, though they keep the aphelion fixed at R.A. $=60^{\circ}\left(\beta=62^{\circ}\right)$ instead of marginalizing over it. When a black curve is below the bluest curve, then all pixels at that longitude are deep enough to detect it. When the black curve is above the reddest curve, then no pixels at that longitude are deep enough to detect it. And when the black curve crosses the $70 \%$ quantile (orange), then $70 \%$ of the pixels are too shallow to detect it. The top and bottom panels are for a $5 M_{\oplus}$ (low: $a=350$ au, $e=0.1$; high: $a=550 \mathrm{au}$, $e=0.3$ ) and $10 M_{\oplus}$ (low: $a=650 \mathrm{au}, e=0.3$; high: $a=850 \mathrm{au}, e=0.5$ ) Planet 9, respectively.

ACT + SO data set, we can expect to detect a $5 M_{\oplus}$ Planet 9 at 500-600 au near the expected aphelion location and 500-900 au over most of the rest of its orbit. This is still not enough to guarantee a discovery, but it will probe a substantial fraction of 


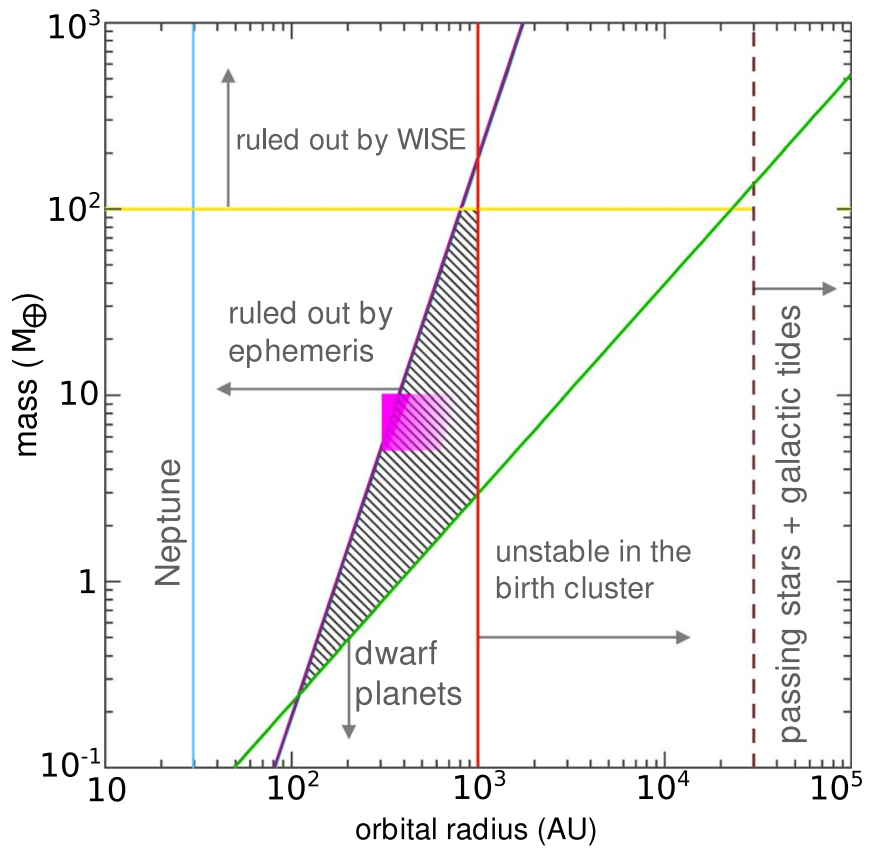

Figure 10. The mass-distance parameter space for undiscovered planets in the solar system with ACT's bounds on Planet 9 shown in magenta. The solid magenta region corresponds to ACT's bounds in its shallowest regions, with the color fading out toward the bounds in its deepest parts. This comes with several caveats: 1 . our bounds are for the current distance, not semimajor axis; 2. only eccentricities relevant for Planet 9 were searched; 3 . masses below $5 M_{\oplus}$ or above $10 M_{\oplus}$ were not searched; 4. distances below 300 au were not searched; 5. distances assume the nominal atmospheric composition; and 6. ACT only covers $40 \%$ of the sky. Figure adapted from B19. Simons Observatory will be more uniform and reach roughly $50 \%$ further.

its parameter space. Unlike bounds from optical surveys like Pan-STARRS and LSST, and even submillimeter ones like WISE, the ACT and SO searches are only mildly sensitive to Planet 9's physical composition, and are robust to assumptions about atmospheric emission lines and albedo.

This work was supported by the U.S. National Science Foundation through awards AST-0408698, AST-0965625, and AST-1440226 for the ACT project, as well as awards PHY0355328, PHY-0855887, and PHY-1214379. Funding was also provided by Princeton University, the University of Pennsylvania, and a Canada Foundation for Innovation (CFI) award to UBC. ACT operates in the Parque Astronómico Atacama in northern Chile under the auspices of the Comisión Nacional de Investigación (CONICYT). Flatiron Institute is supported by the Simons Foundation.

Computations were performed using Princeton Research Computing resources at Princeton University, the Niagara supercomputer at the SciNet HPC Consortium, and the SimonsPopeye cluster of the Flatiron Institute. SciNet is funded by the CFI under the auspices of Compute Canada, the Government of Ontario, the Ontario Research Fund-Research Excellence, and the University of Toronto.

S.N. thanks Bruce Partridge for extensive comments. E.C. acknowledges support from the STFC Ernest Rutherford Fellowship ST/M004856/2 and STFC Consolidated Grant ST/S00033X/1, and from the European Research Council (ERC) under the European Union's Horizon 2020 research and innovation program (grant agreement No. 849169). Research at Perimeter Institute is supported in part by the Government of
Canada through the Department of Innovation, Science and Industry Canada and by the Province of Ontario through the Ministry of Colleges and Universities. S.K.C. acknowledges support from NSF award AST-2001866. K.M.H. is supported by NSF through AST 1815887. N.S., D.H., and A.M. acknowledge support from NSF grant No. AST-1907657.

We gratefully acknowledge the many publicly available software packages that were essential for parts of this analysis. They include healpy (Zonca et al. 2019), HEALPix (Górski et al. 2005), and pixell. ${ }^{55}$ This research made use of Astropy, ${ }^{56}$ a community-developed core Python package for Astronomy (Astropy Collaboration 2013; Price-Whelan et al. 2018). We also acknowledge use of the matplotlib (Hunter 2007) package and the Python Image Library for producing plots in this paper.

\section{Appendix A Smearing \\ A.1. Circular Smearing}

Consider a Gaussian beam with standard deviation $\sigma$, such that its profile is

$$
b(r)=e^{-\frac{1}{2} \frac{r^{2}}{\sigma^{2}}}=e^{-\frac{1}{2} \frac{x^{2}+y^{2}}{\sigma^{2}}}
$$

where $r=\sqrt{x^{2}+y^{2}}$. Partially uncorrected parallax smears this beam along an ellipse with some semimajor axis $\mu$. The simplest and worst case of this is smearing along a circle with radius $\mu$, so that is what we will consider here. This results in the smeared beam

$$
\begin{aligned}
& b_{2}(r, \mu)=\frac{1}{2 \pi} \int_{0}^{2 \pi} d \theta e^{-\frac{1}{2} \frac{(x-\mu \cos \theta)^{2}+(y-\mu \sin \theta)^{2}}{\sigma^{2}}} \\
& \propto b(r) \int_{0}^{2 \pi} d \theta e^{\frac{x \mu \cos \theta+y \mu \sin \theta}{\sigma^{2}}} \\
& \approx b(r) \int_{0}^{2 \pi} d \theta\left(1+\frac{x \mu \cos \theta+y \mu \sin \theta}{\sigma^{2}}\right. \\
& \left.+\frac{1}{2}\left(\frac{x \mu \cos \theta+y \mu \sin \theta}{\sigma^{2}}\right)^{2}\right) \\
& \propto b(r)\left(1+\frac{1}{4} \frac{r^{2} \mu^{2}}{\sigma^{4}}\right)
\end{aligned}
$$

where we have assumed $\mu \ll \sigma$ and have ignored any factors that just scale the overall amplitude of the function. If all values $\mu \in\left[-\frac{\Delta}{2}, \frac{\Delta}{2}\right]$ occur with equal weight, then the average beam across all of these will be:

$$
\begin{aligned}
& b_{\mathrm{eff}}(r)=\frac{1}{\Delta} \int_{-\frac{\Delta}{2}}^{\frac{\Delta}{2}} d \mu b_{2}(r, \mu)=\frac{1}{\Delta} b(r) \int_{-\frac{\Delta}{2}}^{\frac{\Delta}{2}} d \mu \\
& \times\left(1+\frac{1}{4} \frac{r^{2} \mu^{2}}{\sigma^{4}}\right)=b(r)\left(1+\frac{1}{24} \frac{r^{2} \Delta^{2}}{\sigma^{4}}\right) \\
& \approx e^{-\frac{1}{2} \frac{r^{2}}{\sigma^{2}}} e^{\frac{1}{24} \frac{r^{2} \Delta^{2}}{\sigma^{4}}}=e^{-\frac{1}{2} \frac{r^{2}}{\sigma_{\mathrm{eff}}^{2}}}
\end{aligned}
$$

\footnotetext{
55 https://github.com/simonsobs/pixell

56 http://www.astropy.org
} 

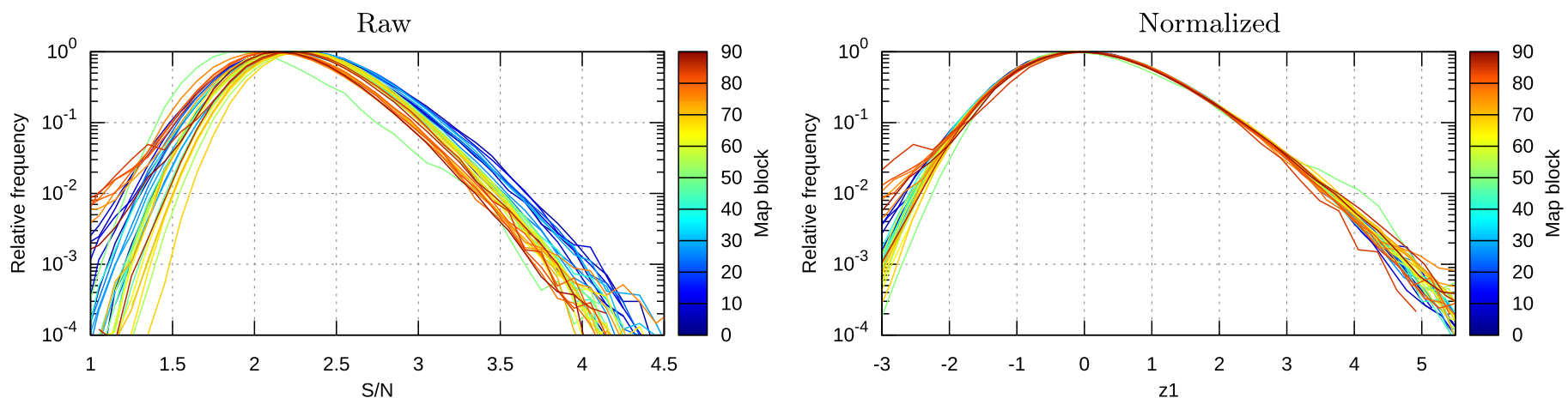

Figure 11. Spatial normalization of the detection statistic. Left: histograms for the shift-and-stack $\mathrm{S} / \mathrm{N}$ ratio for the 300 au case. Each curve corresponds to a different $5^{\circ} \times 5^{\circ}$ part of the map. The distribution is non-Gaussian and spatially variable. Right: the same histograms after normalizing by subtracting the mean and dividing by the standard deviation. After this the distribution is no longer spatially variable. The actual spatial normalization used in the search uses smaller $0.5 \times 4^{\circ}$ degree tiles, which are large enough to measure the mean and standard deviation reliably, but result in noisier histograms.

where

$$
\begin{aligned}
\sigma_{\mathrm{eff}}^{2} & =\frac{\sigma^{2}}{1-\frac{1}{12} \frac{\Delta^{2}}{\sigma^{2}}} \approx \sigma^{2}+\frac{1}{12} \Delta^{2} \\
\Leftrightarrow \mathrm{FWHM}_{\mathrm{eff}}^{2} & =\mathrm{FWHM}^{2}+\frac{2 \log (2)}{3} \Delta^{2} .
\end{aligned}
$$

So circular smearing adds in quadrature to the beam size.

\section{A.2. Linear Smearing}

We here smear the beam linearly in the $x$ direction with $\mu \in\left[-\frac{\Delta}{2}, \frac{\Delta}{2}\right]$.

$$
\begin{gathered}
b_{\text {eff }}=\frac{1}{\Delta} \int_{-\frac{\Delta}{2}}^{\frac{\Delta}{2}} d \mu^{-\frac{1}{2} \frac{(x-\mu)^{2}+y^{2}}{\sigma^{2}}} \approx b(r) \int_{-\frac{\Delta}{2}}^{\frac{\Delta}{2}} d \mu \\
\left(1+\frac{x \mu}{\sigma^{2}}+\frac{1}{2} \frac{x^{2} \mu^{2}}{\sigma^{4}}\right) \frac{e^{-\frac{1}{2} \frac{\mu^{2}}{\sigma^{2}}}}{\Delta} \\
\propto b(r)\left(1+\frac{1}{12} \frac{x^{2} \Delta^{2}}{\sigma^{4}}\right) \approx e^{-\frac{1}{2}\left(\frac{x^{2}}{\sigma_{x, \text { eff }}}+\frac{y^{2}}{\sigma^{2}}\right)}
\end{gathered}
$$

with $\sigma_{x \text {,eff }}^{2}=\sigma^{2}+\frac{1}{6} \Delta^{2}$. Since only the $x$ direction was smeared, the beam is now slightly elliptical. For the purposes of $\mathrm{S} / \mathrm{N}$, what matters is not the shape of the beam, but its area,

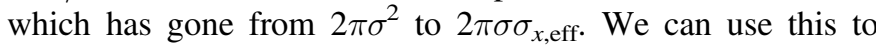
define an effective overall beam standard deviation:

$$
\begin{gathered}
\sigma_{\text {eff }}^{2}=\sigma \sigma_{x, \text { eff }}=\sigma^{2} \sqrt{1+\frac{1}{6} \frac{\Delta^{2}}{\sigma^{2}}} \approx \sigma^{2}+\frac{1}{12} \Delta^{2} \\
\Leftrightarrow \mathrm{FWHM}_{\mathrm{eff}}^{2}=\mathrm{FWHM}^{2}+\frac{2 \log (2)}{3} \Delta^{2}
\end{gathered}
$$

which happens to be the same result as what we got for circular smearing.

\section{Appendix B Building the Detection Statistic $z$}

The $\mathrm{S} / \mathrm{N}$ map produced by the shift-and-stack search is nonGaussian with properties that depend on both the distance $r$ and the position in the map, making it unsuitable as an indication of the detection strength. However, we found that the following three-step approach allowed us to transform $\mathrm{S} / \mathrm{N}$ into a much more well-behaved detection statistic $z$.

\section{B.1. Spatial Normalization}

For each $r$ we measure the mean $\mu_{\text {spat }}(r)$ and standard deviation $\sigma_{\text {spat }}(r)$ of the $\mathrm{S} / \mathrm{N}$ map as a function of position. ${ }^{57}$ Using these, we define the spatially normalized detection statistic $z_{1}$ as

$$
z_{1}(r)=\left[\mathrm{S} / \mathrm{N}(r)-\mu_{\text {spat }}(r)\right] / \sigma_{\text {spat }}(r) .
$$

This normalization process is shown in Figure 11.

\section{B.2. Distance Normalization}

We then build the empirical survival function $N\left(z_{1}>x\right)$ for all peaks in the tile with $z_{1}>1$ and map this to a corresponding Gaussian quantile

$$
z^{*}=-\sqrt{2} \operatorname{erf}^{-1}(2 N / n-1) .
$$

The value of $n$ controls how far into the tail of a Gaussian survival function we map our empirical survival function. Its exact value is not important as long as $n>\max (N)$. It should be kept constant for all values of $r$ to ensure that equally rare values of $z_{1}$ map to the same $z^{*}$ for all distances. We chose $n=A_{\text {tile }} / A_{\text {peak }} \approx\left(12^{\circ} / 2^{\prime}\right)^{2}=1.3 \cdot 10^{6}$, with $A_{\text {tile }}=\left(12^{\circ}\right)^{2}$ being the area of the tile, and $A_{\text {peak }}=\left(2^{\prime}\right)^{2}$ being the approximate feature size in the $z_{1}$ map. In principle Equation (B8) could be used to directly normalize $z_{1}$, but in practice there are too few samples in the tail. However, $z^{*}$ turns out to be very well approximated as a linear function of $z_{1}{ }^{58}$ We use this to define the fully normalized detection statistic

$$
z(r)=\left[z_{1}(r)-\mu_{\text {dist }}(r)\right] / \sigma_{\text {dist }}(r)
$$

where $\mu_{\text {dist }}$ and $\sigma_{\text {dist }}$ are the offset and slope of the function $z^{*}\left(z_{1}\right)$. This process is illustrated in Figure 12. Inserting the expression

\footnotetext{
57 We do this in $0.5 \times 4^{\circ}$ blocks. These short-wide blocks were chosen because many features in the ACT exposure pattern are wider than they are tall. The block size is a compromise between angular resolution and sample variance in the estimates.

58 This means that the upper tail of the $z_{1}$ distribution (which is what we care about for feature detection) is nearly Gaussian, even though the whole distribution is not.
} 

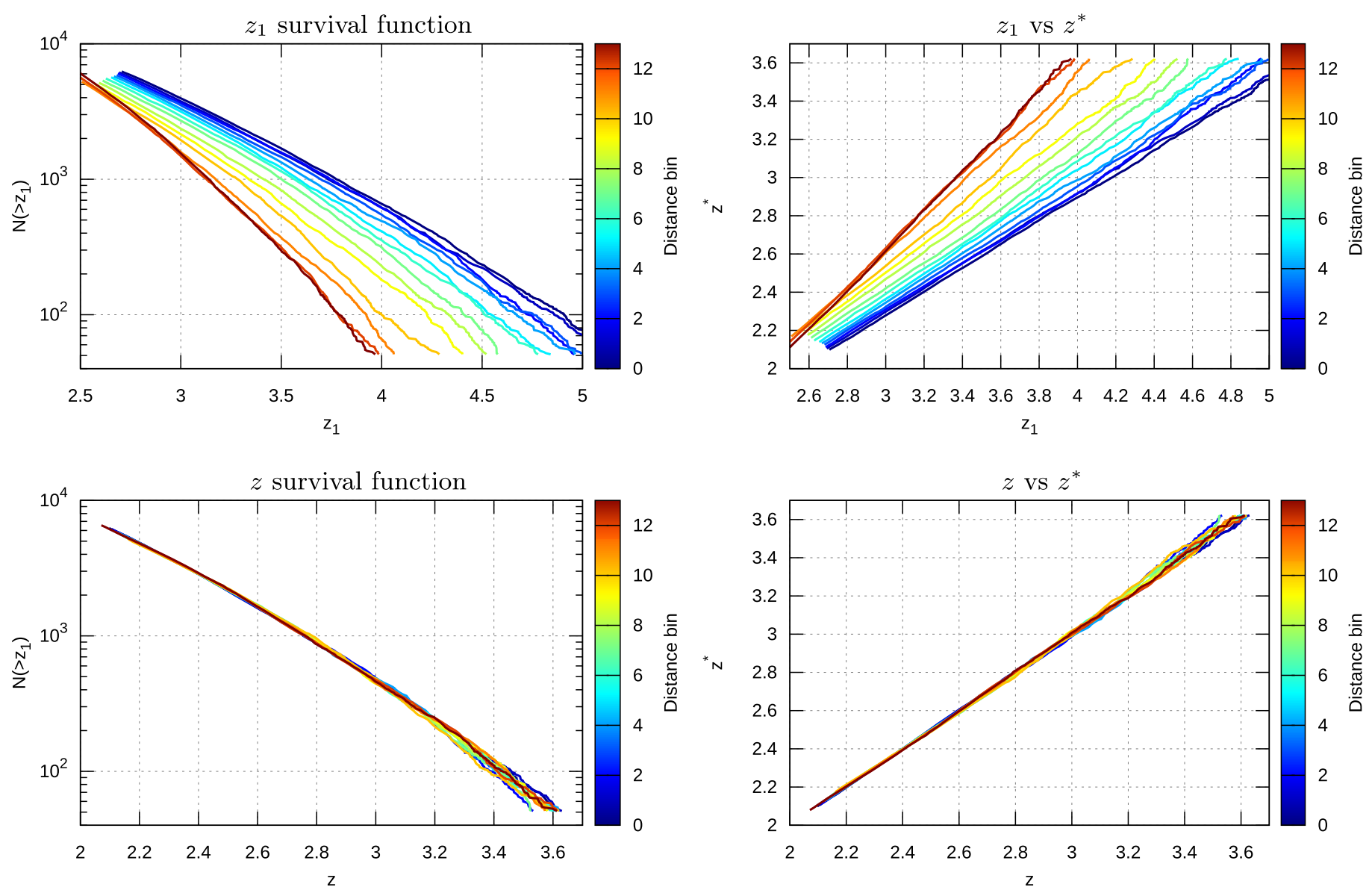

Figure 12. Distance normalization of the detection statistic. Top left: the tail of the survival function of $z_{1}$. Each color corresponds to a different distance bin, from 300 au (blue) to $2000 \mathrm{au}$ (red). The different distances clearly do not follow the same distribution. Top right: as top left, but with the survival function replaced with the corresponding Gaussian quantile $z$. For each distance we define $\mu_{\text {dist }}$ and $\sigma_{\text {dist }}$ as the best-fit linear offset and slope of its curve. Bottom left: the survival function for the distance-normalized detection statistic $z=\left(z_{1}-\mu_{\text {dist }}\right) / \sigma_{\text {dist }}$. All distances now follow the same distribution. Bottom right: $z$ vs. the empirical Gaussian quantiles $z$. They are practically identical.

for $z_{1}$ into Equation (B9), we get the full normalization

$$
z(r)=\left(\mathrm{S} / \mathrm{N}(r)-\mu_{z}(r)\right) / \sigma_{z}(r)=\xi(\mathrm{S} / \mathrm{N})
$$

where we have defined $\mu_{z}=\mu_{\text {spat }}+\sigma_{\text {spat }} \mu_{\text {dist }}$ and $\sigma_{z}=$ $\sigma_{\text {spat }} \sigma_{\text {dist }}$; and we have implicitly defined the function $\xi$.

\section{Appendix C \\ Ad-hoc Filter}

The map noise power can be approximately modeled as $1 / \beta$ $\left(\ell, \ell_{\text {knee }}, \alpha\right)$, where $\beta$ is the Butterworth filter profile

$$
\beta\left(\ell, \ell_{\text {knee }}, \alpha\right)=1 /\left[1+\left(\ell / \ell_{\text {knee }}\right)^{\alpha}\right]
$$

This noise power spectrum takes the form of a power law with slope $\alpha \approx-4$ at low $\ell$ (mainly caused by atmospheric emission) which transitions to a flat "noise floor" around the multipole $\ell_{\text {knee }} \approx 3000$ where photon noise and detector readout noise start to dominate. ${ }^{59}$ However, it turned out that $N$, the noise model we use for our time-ordered data analysis (see Equation (5)), does not capture the full correlation structure of the atmosphere, and ends

\footnotetext{
59 Multipole $\ell_{\text {knee }}$ is frequency dependent, taking values of about 2000/3000/ 4000 at $\mathrm{f} 090 / \mathrm{f} 150 / \mathrm{f} 220$, but because this issue was discovered after the frequency maps had already been combined we will just use a representative 3000 here.
}

up underestimating the effective $\ell_{\text {knee }}$ by a factor of two, i.e., $\ell_{\text {knee }}{ }^{\prime} \approx 1500$. This means that our matched filter did not suppress noise in the $1500 \lesssim \ell \lesssim 3000$ range as much as it should, leading to a loss in $\mathrm{S} / \mathrm{N}$.

To correct for this, we replace the map inverse covariance matrix $U^{-1}$ with $\beta(\ell, 3000,-4) U^{-1}$. Accordingly $\rho=R^{T} U^{-1} \hat{m}$ is remapped at $\rho \rightarrow \beta \rho$. What happens to $\kappa=\operatorname{diag}\left(R^{T} U^{-1} R\right)$ is harder to estimate. We can approximate it as $\kappa \rightarrow q \kappa$, where $q$ a single number representing the weighted average of the extra filter $\beta(\ell, 3000,-4)$ over all multipoles,

$$
q=\frac{\sum_{\ell}(2 \ell+1) W(\ell) \beta(\ell, 3000,-4)}{\sum_{\ell}(2 \ell+1) W_{\ell}}
$$

with the weights $W(\ell)$ being a harmonic-space approximation of the original matched filter,

$$
W(\ell)=\beta(\ell, 1500,-4) B(\ell)
$$

where $B(\ell)$ is the beam and $\beta(\ell, 1500,-4)$ approximates the original $U^{-1}$. However, in the end the normalization of $\kappa$ does not matter, since it is absorbed by the simulation-based debiasing we do to account for the effect of mean sky subtraction in Section 4.6. 
The ad-hoc filter could have been avoided if we had computed the full $\hat{m}$ and had done the full matched filter in pixel space instead of using the "rhs" computational shortcut described in Section 4.3.4. This is a potential improvement for future analyses.

\section{Appendix D ACT Data Set Details}

Table 5 summarizes the ACT data sets used in this analysis.

Table 5

ACT Data Sets Used in the Analysis

\begin{tabular}{lcccl}
\hline \hline Survey & Patch & R.A. (deg) & Decl. (deg) & Data Sets \\
\hline ACT DR4 & D1 & 140 to 161 & -5 to 6 & PA1 2013 \\
ACT DR4 & D5 & -19 to 13 & -7 to 6 & PA1 2013 \\
ACT DR4 & D6 & 19 to 48 & -11 to 1 & PA1 2013 \\
ACT DR4 & D56 & -23 to 54 & -10 to 7 & PA1+PA2 2014-2015, PA3 2015 \\
ACT DR4 & D8 & -12 to 18 & -52 to -32 & PA1+PA2+PA3 2015 \\
ACT DR4 & BN & 102 to 257 & -7 to 22 & PA1+PA2+PA3 2015 \\
ACT DR4 & AA & 0 to 360 & -62 to 22 & PA2+PA3 2016 \\
AdvACT & AA & 0 to 360 & -62 to 22 & PA4+PA5+PA6 2017-2019 \\
ACT day & BN & 102 to 257 & 3 to 20 & PA1+PA2 2014-2015, PA3 2015 \\
ACT day & Day-N & 162 to 258 & -52 to -29 & PA2+PA3 2016, PA4+PA5+PA6 2017-2019 \\
ACT day & Day-S & -25 to 60 & \\
\hline
\end{tabular}

Note. They are identical to those used in ACT DR5 (Naess et al. 2020), except for the inclusion of data from the 2019 observing season, and the exclusion of Planck (too low resolution) and ACT MBAC (too low sky coverage). PA $\{1-6\}$ refers to the individual detector arrays in the instrument, with PA $\{1,2\}$ covering the f150 band, PA $\{3,5,6\}$ covering f090 and f150, and PA4 covering f150 and f220.

\section{ORCID iDs}

Sigurd Naess (1) https://orcid.org/0000-0002-4478-7111 Simone Aiola (i) https://orcid.org/0000-0002-1035-1854 Nick Battaglia (i) https://orcid.org/0000-0001-5846-0411 Richard J. Bond (i) https://orcid.org/0000-0003-2358-9949 Erminia Calabrese (ㄴ) https://orcid.org/0000-0003-0837-0068 Steve K. Choi 1 (1) htps://orcid.org/0000-0002-9113-7058 Nicholas F. Cothard (ii) https://orcid.org/0000-0002-6151-6292 Mark Halpern (10 https://orcid.org/0000-0002-1760-0868 J. Colin Hill (1) https://orcid.org/0000-0002-9539-0835 Brian J. Koopman (ii) https://orcid.org/0000-0003-0744-2808 Mark Devlin (i) https://orcid.org/0000-0002-3169-9761 Jeff McMahon (1) https://orcid.org/0000-0002-7245-4541 Simon Dicker @ ittps://orcid.org/0000-0002-1940-4289 Adriaan J. Duivenvoorden (1) https://orcid.org/0000-00032856-2382

Jo Dunkley (i) https://orcid.org/0000-0002-7450-2586

Valentina Fanfani (i) https://orcid.org/0000-00032410-0922

Simone Ferraro (1) https://orcid.org/0000-0003-4992-7854

Patricio A. Gallardo (1) https://orcid.org/0000-0001-

9731-3617

Yilun Guan (1) https://orcid.org/0000-0002-1697-3080

Dongwon Han (i) https://orcid.org/0000-0001-5649-3551

Matthew Hasselfield (i) https://orcid.org/0000-00022408-9201

Adam D. Hincks 나 https://orcid.org/0000-0003-1690-6678

Kevin Huffenberger (1) https://orcid.org/0000-00017109-0099

Arthur B. Kosowsky 1 https://orcid.org/0000-00023734-331X

Thibaut Louis (1) https://orcid.org/0000-0002-6849-4217
Mathew S. Madhavacheril (1) https://orcid.org/0000-00016740-5350

Federico Nati (1) https://orcid.org/0000-0002-8307-5088

Michael D. Niemack (i) https://orcid.org/0000-00017125-3580

Lyman Page (1) https://orcid.org/0000-0002-9828-3525

Maria Salatino (1) https://orcid.org/0000-0003-4006-1134

Emmanuel Schaan (1) https://orcid.org/0000-0002-4619-8927

John Orlowski-Scherer (i) https://orcid.org/0000-00031842-8104

Alessandro Schillaci (1) https://orcid.org/0000-0002-

0512-1042

Neelima Sehgal iㅣ https://orcid.org/0000-0002-9674-4527

Cristóbal Sifón (1) https://orcid.org/0000-0002-8149-1352

Suzanne Staggs (10 https://orcid.org/0000-0002-7020-7301

Edward J. Wollack (i) https://orcid.org/0000-0002-7567-4451

\section{References}

AllWISE 2013, AllWISE explanatory supplement, https://wise2.ipac.caltech. edu/docs/release/allwise/expsup/sec2_1.html

Astropy Collaboration 2013, A\&A, 558, A33

Batygin, K., Adams, F. C., Brown, M. E., \& Becker, J. C. 2019, PhR , 805, 1

Batygin, K., \& Brown, M. E. 2016, AJ, 151, 22

Batygin, K., \& Brown, M. E. 2021, ApJL, 910, L20

Baxter, E. J., Jain, B., Blake, C., et al. 2018, arXiv:1812.08701

Bernardinelli, P. H., Bernstein, G. M., Sako, M., et al. 2020, PSJ, 1, 28 Bernstein, G. M., Trilling, D. E., Allen, R. L., et al. 2004, AJ, 128, 1364

Carlstrom, J. E., Ade, P. A. R., Aird, K. A., et al. 2011, PASP, 123, 568 Chambers, K. C., et al. 2016, arXiv:1612.05560

Choi, S. K., Austermann, J., Basu, K., et al. 2020, JLTP, 199, 1089

Choi, S. K., Austermann, J., Beall, J. A., et al. 2018, JLTP, 193, 267

Cowan, N. B., Holder, G., \& Kaib, N. A. 2016, ApJL, 822, L2

Fienga, A., Di Ruscio, A., Bernus, L., et al. 2020, A\&A, 640, A6 
Fortney, J. J., Marley, M. S., Laughlin, G., et al. 2016, ApJL, 824, L25

Fowler, J. W., Niemack, M. D., Dicker, S. R., et al. 2007, ApOpt, 46 3444

Gladman, B., Kavelaars, J. J., Nicholson, P. D., Loredo, T. J., \& Burns, J. A. 1998, AJ, 116, 2042

Górski, K. M., Hivon, E., Banday, A. J., et al. 2005, ApJ, 622, 759

Ho, S.-P. P., Austermann, J., Beall, J. A., et al. 2017, Proc. SPIE, 9914, 991418

Holman, M. J., Payne, M. J., Blankley, P., Janssen, R., \& Kuindersma, S. 2018, arXiv: 1805.02638

Hunter, J. D. 2007, CSE, 9, 90

Ishihara, D., Onaka, T., Kataza, H., et al. 2010, A\&A, 514, A1

Ivezić, Ž., Kahn, S. M., Tyson, A. J., et al. 2019, ApJ, 873, 111

Joint IRAS Science Working Group 1986, IRAS Catalog of Point Sources, v2.0, https://heasarc.gsfc.nasa.gov/W3Browse/iras/iraspsc.html

Linder, E. F., \& Mordasini, C. 2016, A\&A, 589, A134

Luhman, K. L. 2013, ApJ, 781, 4
Meisner, A. M., Bromley, B. C., Kenyon, S. J., \& Anderson, T. E. 2018, AJ, 155,166

Naess, S., Aiola, S., Austermann, J. E., et al. 2020, JCAP, 2020, 046

Naess, S., Battaglia, N., Richard Bond, J., et al. 2021, ApJ, 915, 14

Napier, K. J., Gerdes, D. W., Lin, H. W., et al. 2021, PSJ, 2, 59

Pan-STARRS 2015, The Pan-STARRS Survey for Transients, https://star.pst. qub.ac.uk/ps1threepi/psdb/

Price-Whelan, A. M., Hogg, D. W., Rix, H.-W., et al. 2018, AJ, 156, 123

Schlafly, E. F., Meisner, A. M., \& Green, G. M. 2019, ApJS, 240, 30

Shankman, C., Kavelaars, J. J., Bannister, M. T., et al. 2017, AJ, 154, 50

SO Collaboration 2019, JCAP, 2019, 056

Thornton, R. J., Ade, P. A. R., Aiola, S., et al. 2016, ApJS, 227, 21

Yamamura, I., Makiuti, S., Ikeda, Y., et al. 2010, AKARI/FIS All-Sky Survey Bright Source Catalogue v1.0 Release Note, https://irsa.ipac.caltech.edu/ data/AKARI/documentation/AKARI-FIS_BSC_V1_RN.pdf

Zonca, A., Singer, L., Lenz, D., et al. 2019, JOSS, 4, 1298 